\title{
Image Registration \\ for Remote Sensing
}

\author{
Jacqueline Le Moigne \\ Nathan S. Netanyahu
}

Roger D. Eastman 
A Few Memories, 1983 to 1988 ...

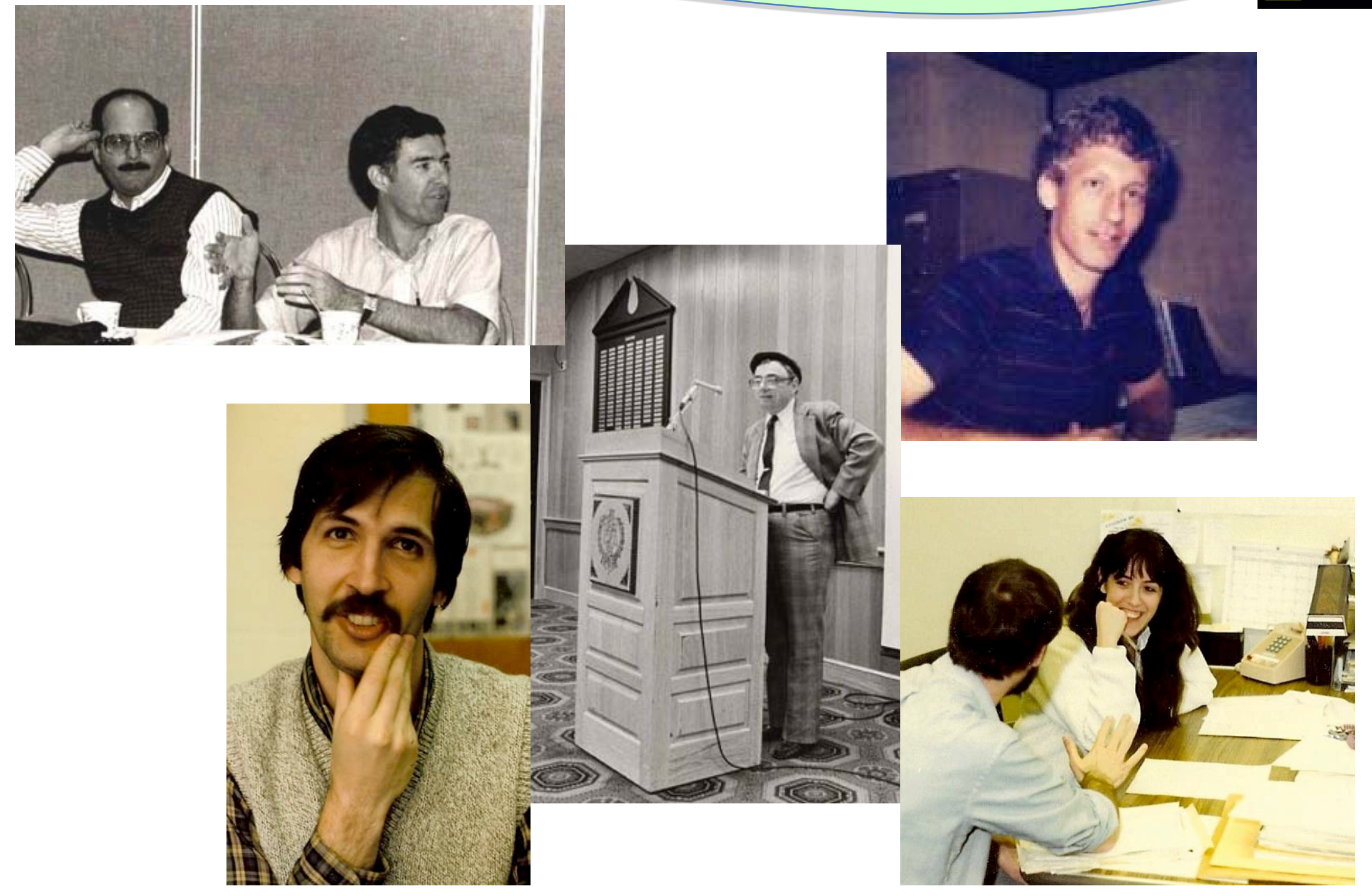




\section{Around CVL, 1983 to 1988 ...}

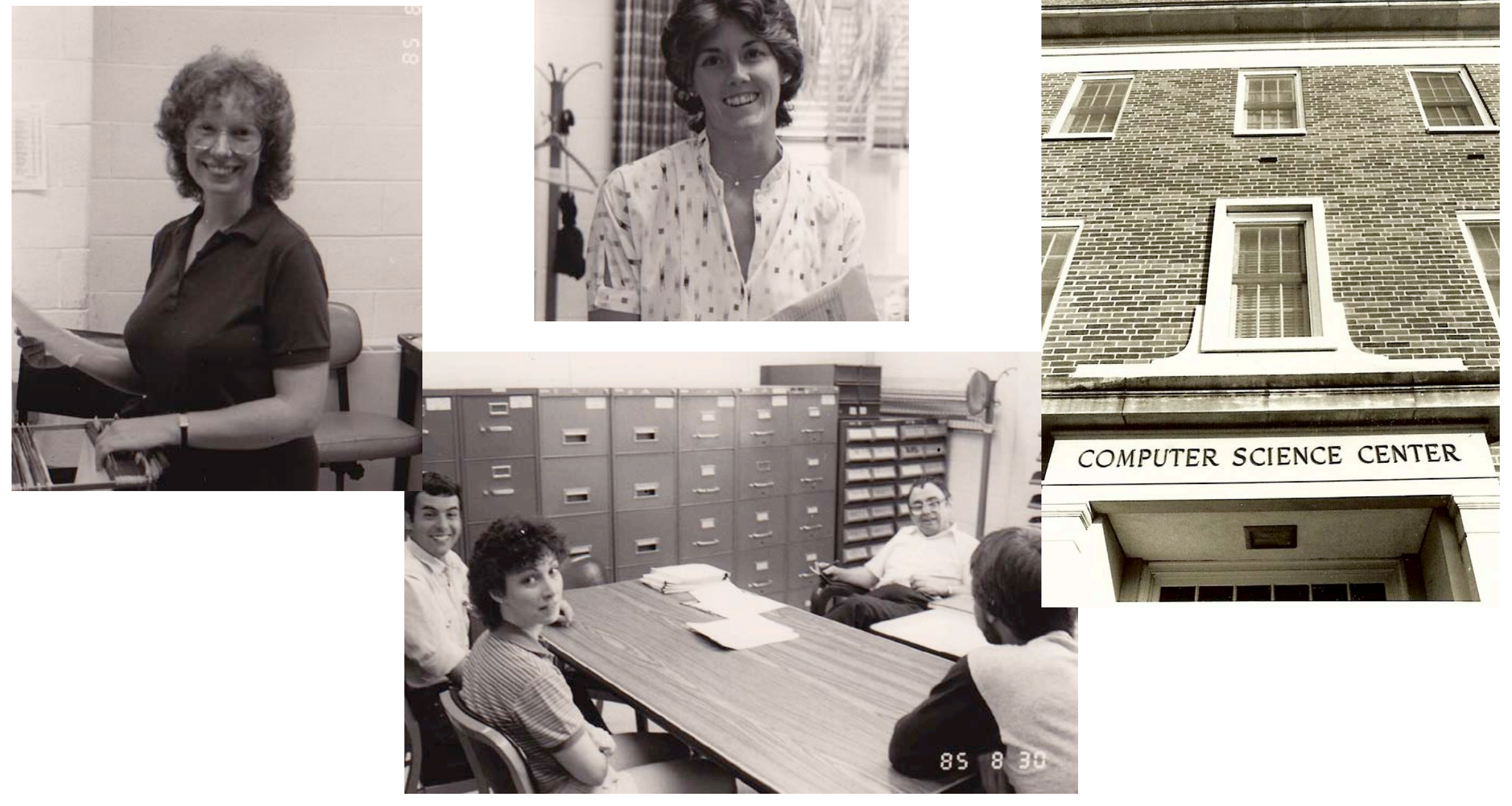




\title{
Context and Background
}

\author{
Jacqueline Le Moigne
}

NASA Goddard Space Flight Center 


\section{Image Registration}

\section{in the Context of Space}

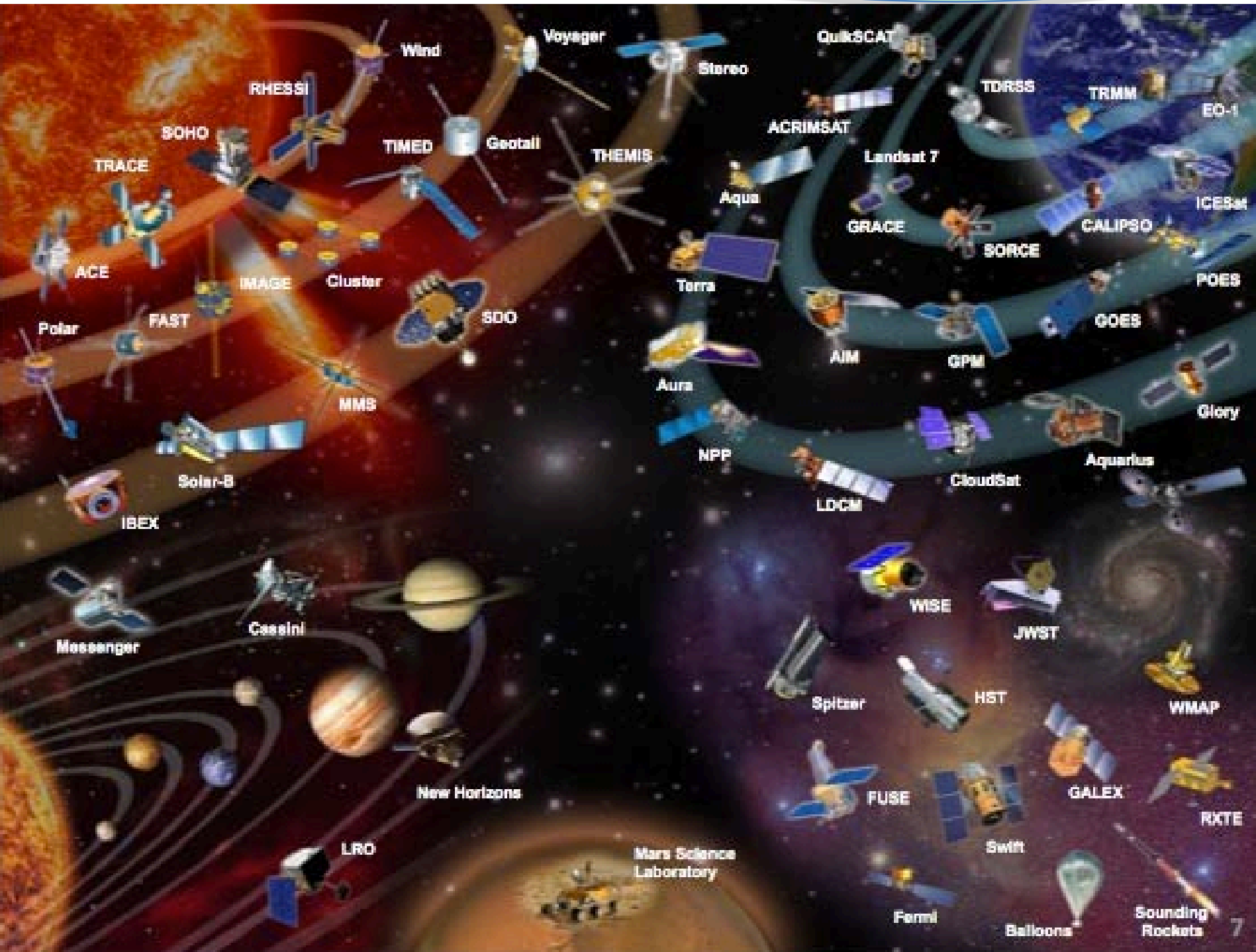




\section{Image Registration}

\section{in the Context of Earth Remotese,}

\section{Earth Science}

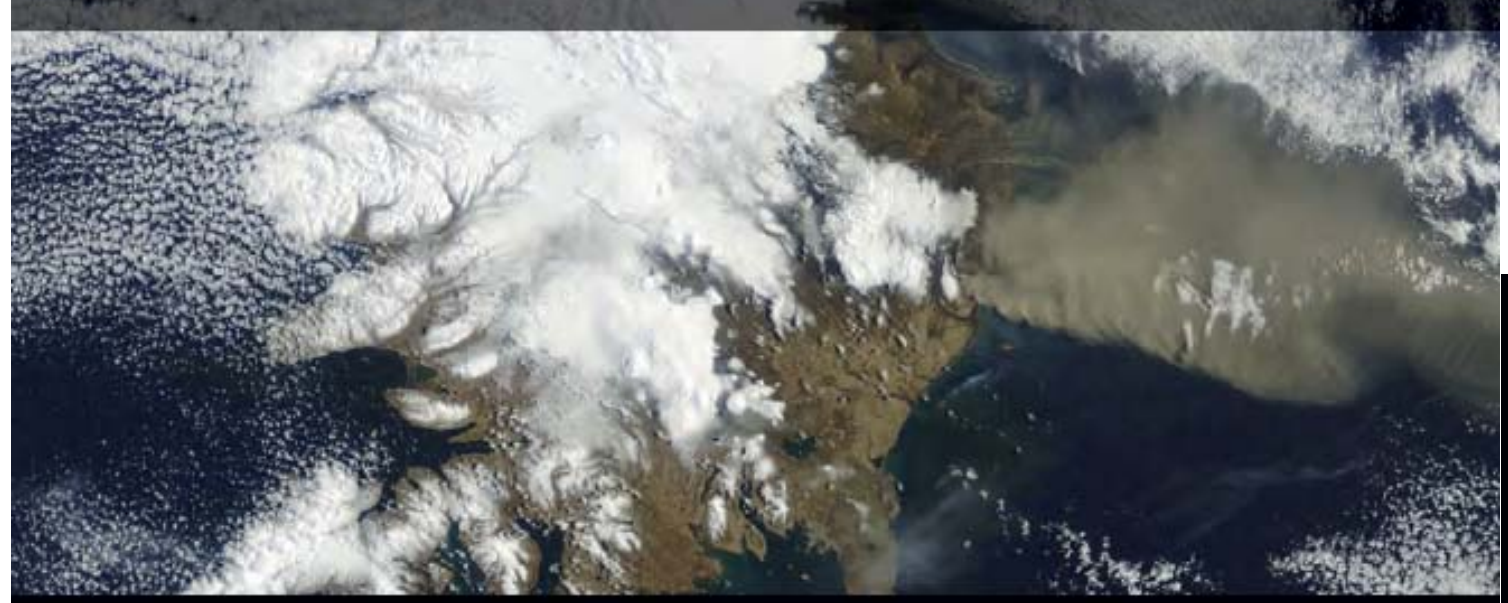

NASA pioneered the interdisciplinary field of Earth System Science - the study of the Earth as an integrated system. This approach to studying the Earth as a single complex system is essential to understanding the causes and consequences of climate change and other global environmental concerns. Spaceborne instruments provide essential broad coverage, high spatial resolution, frequent sampling, and near-uniform accuracy and stability. Multiple on-orbit missions, including those flying in coordinated orbits as part of planned constellations, allow data to be acquired simultaneously on many important quantities, enabling investigations of the interactions among the coupled Earth processes that constitute the climate system. NASA's research, coupled with that of our partners in the U.S. Global Change Research Program, provides much of the nation's knowledge base for understanding, mitigating, and adapting to climate change.

Advance Earth System Science to meet the challenges of climate and environmental change.

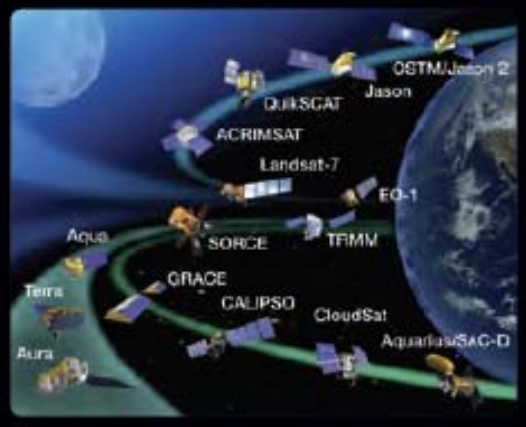




\section{Spatial and Spectral Characteristics}

\section{of Some Operational Sensors (Ch 184-2}

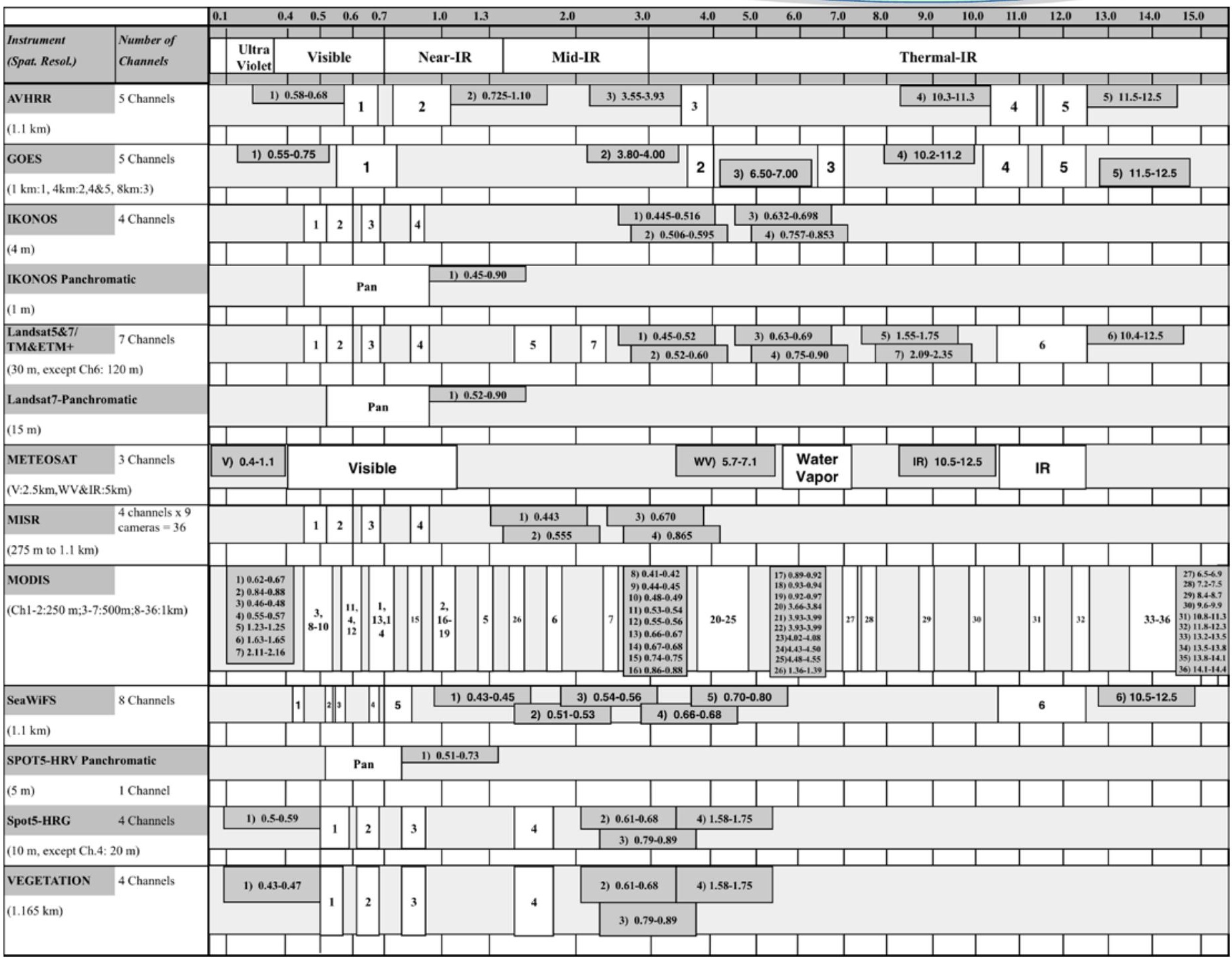


What is Image Registration ...

- Definition

"Exact pixel-to-pixel matching of two different images or matching of one image to a map"

- Multiple Source Data

- Multimodal Registration

- Temporal Registration

- Viewpoint Registration

- Template Registration 


\section{Challenges in Image Registration}

- Remote Sensing vs. Medical or Other Imagery

- Variety in the types of sensor data and the conditions of data acquisition

- Size of the data

- Lack of a known image model

- Lack of well-distributed "fiducial points" resulting in lack of algorithms validation

- Navigation Error

- Atmospheric and Cloud Interactions
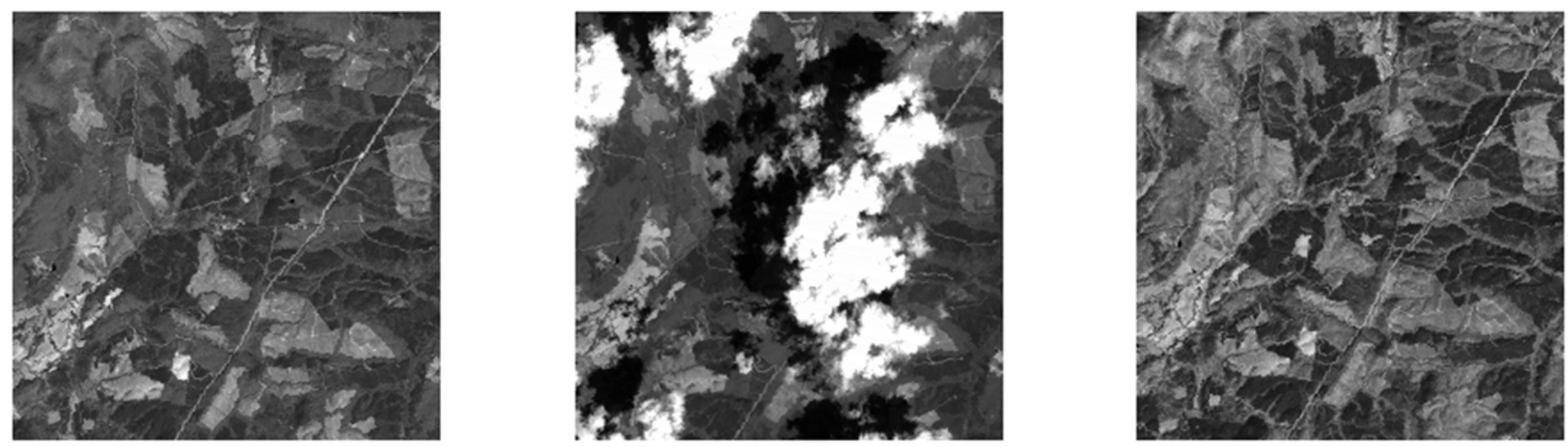

Three Landsat images over Virginia acquired in August, October, and November 1999 (Courtesy: Jeffrey Masek, NASA Goddard Space Flight Center) 


\section{Challenges in Image Registration}

Atmospheric and Cloud Interactions

Baja Peninsula,

California; 4 different times of the day (GOES-8)

(Reproduced from Le Moigne \& Eastman, 2005)

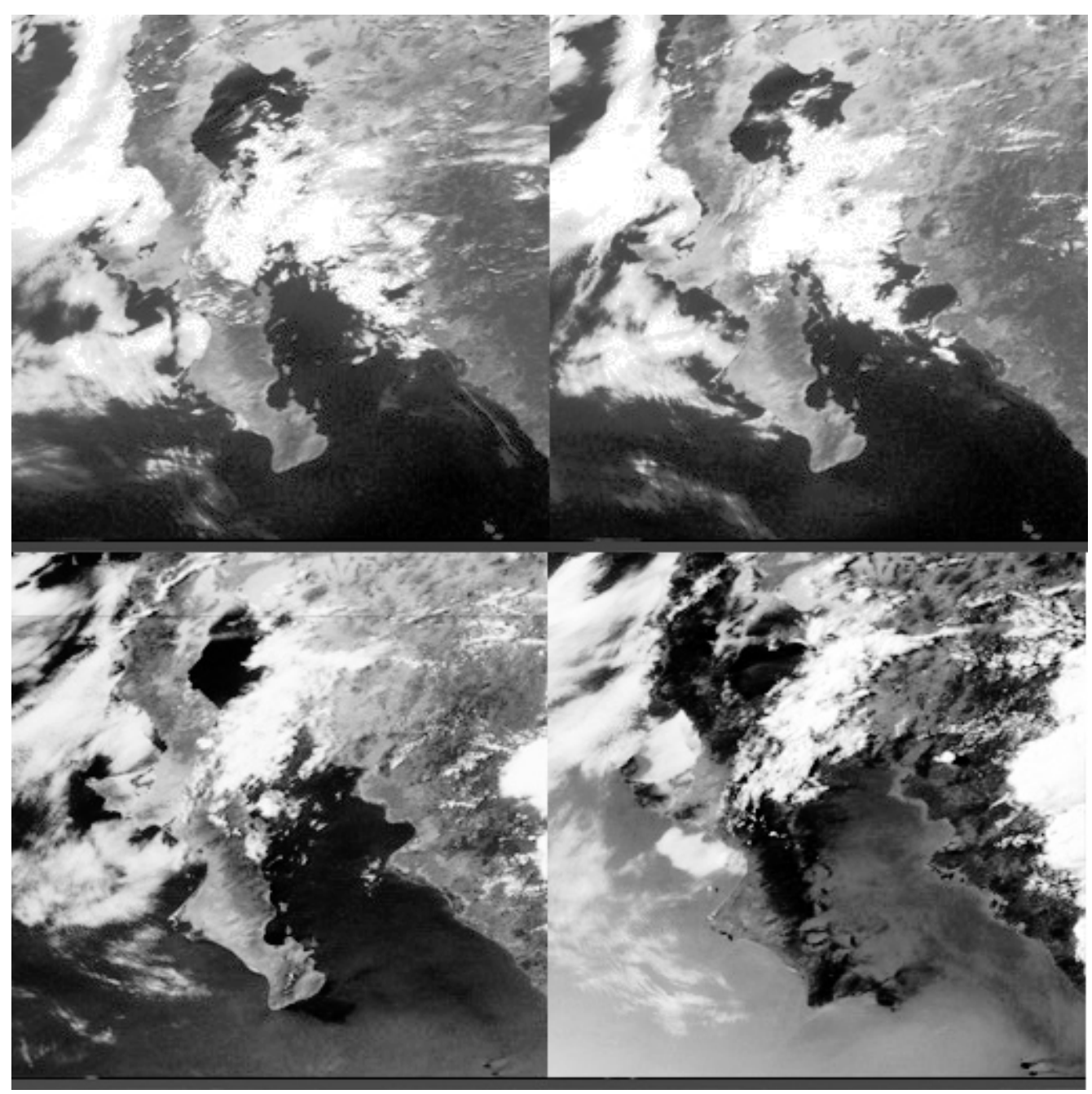




\section{Challenges in Image Registration}

Multitemporal Effects

Mississippi and Ohio Rivers before \& after Flood of Spring 2002

(Terra/MODIS)

(Reproduced from Le Moigne \& Eastman, 2005)
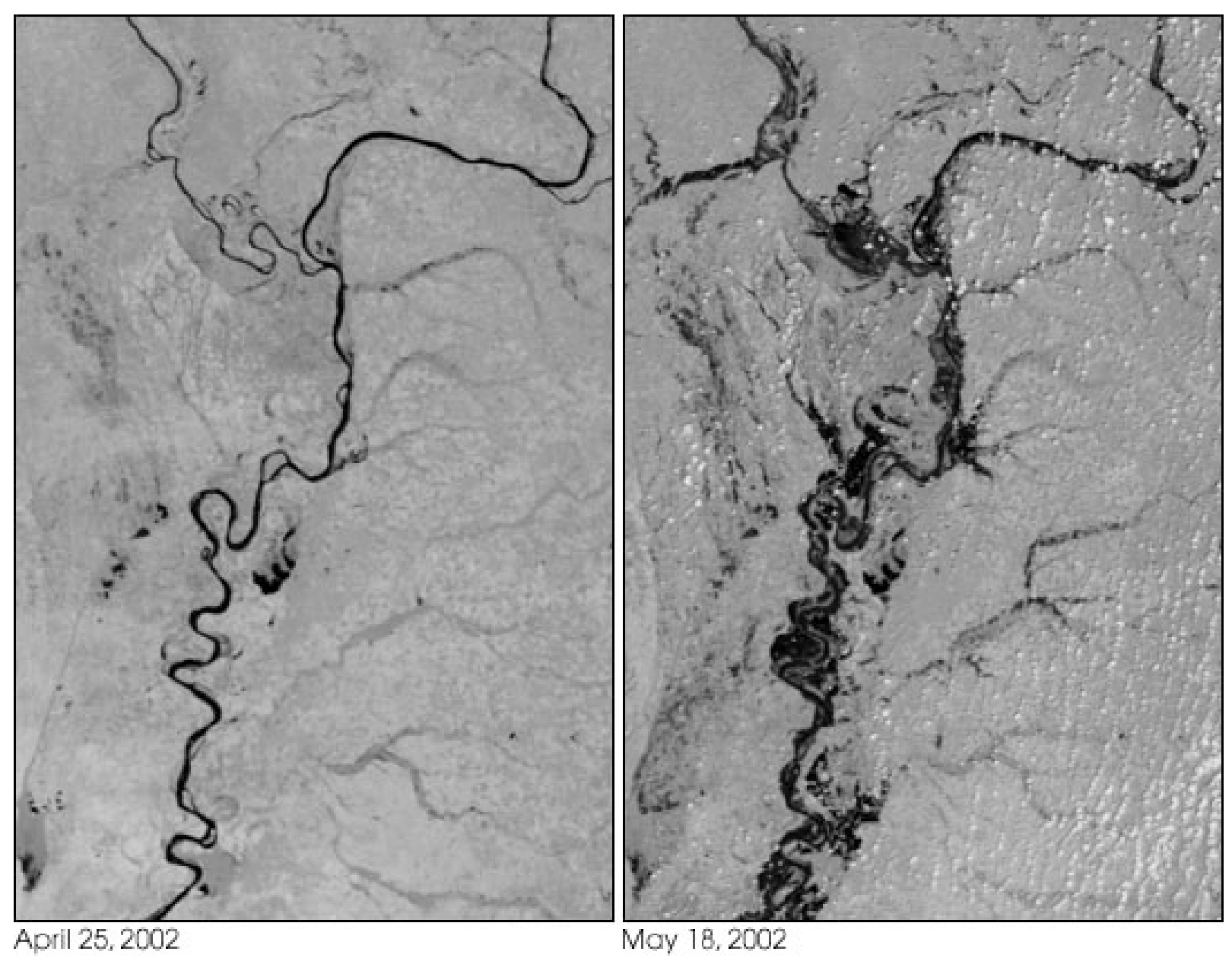

May 18, 2002 


\section{Challenges in Image Registration}

\section{Relief Effect}

SAR and Landsat-TM Data of Lopé Area, Gabon, Africa (Reproduced from Le Moigne \& Eastman, 2005)
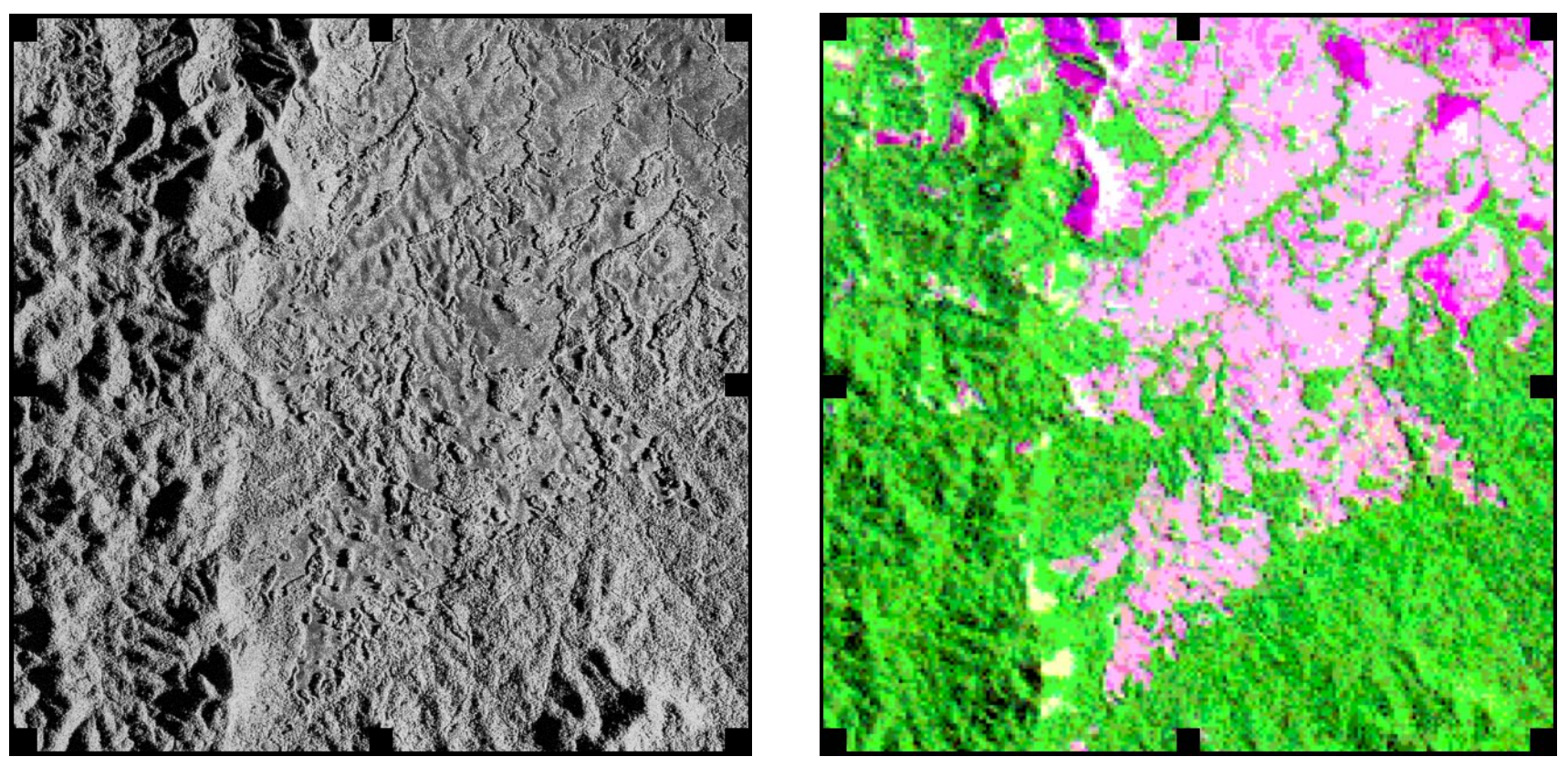


\section{Image Registration or Precision Correction}

- Navigation or Model-Based Systematic Correction

- Orbital, Attitude, Platform/Sensor Geometric Relationship, Sensor Characteristics, Earth Model, etc.

- Image Registration/Feature-Based Precision Correction

- Navigation within a Few Pixels Accuracy

- Image Registration Using Selected Features (or Control Points) to Refine Geo-Location Accuracy

- Image Registration as a Post-Processing or as a Feedback to Navigation Model 


\section{Misregistration}

- (Towsnhend et al, 1992) and (Dai \& Khorram, 1998): small error in registration may have a large impact on global change measurements accuracy

- e.g., 1 pixel misregistration error $=>50 \%$ error in Vegetation Index (NDVI) computation (using 250m MODIS data)
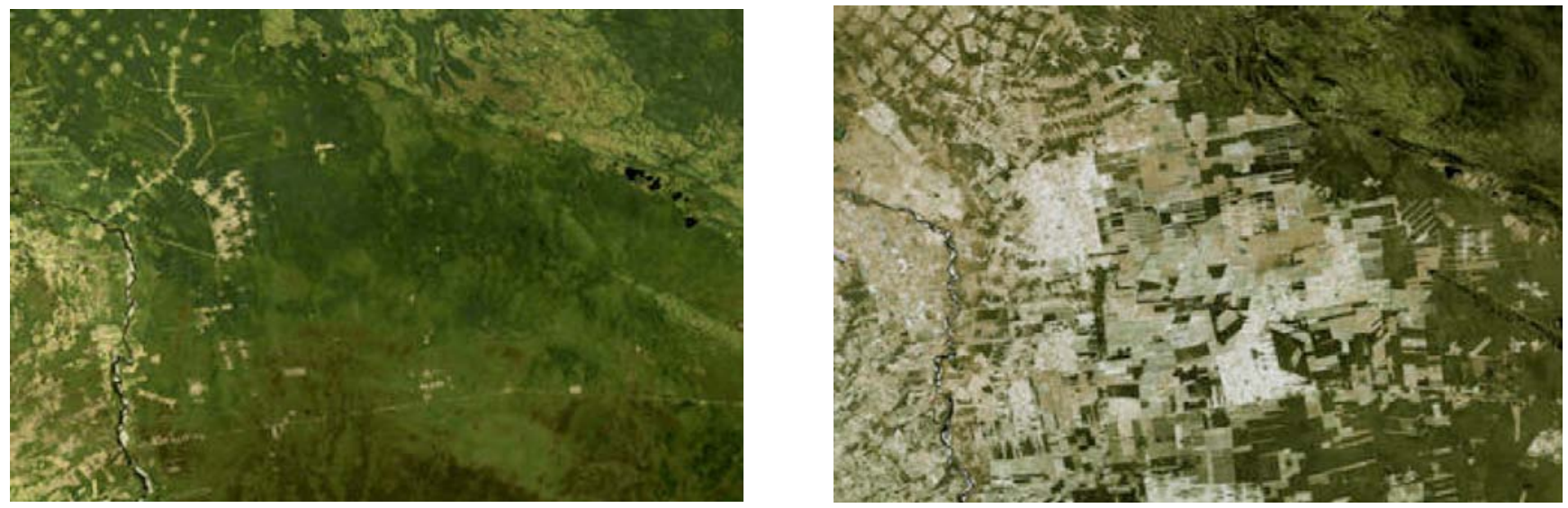

Human-induced land cover changes observed by Landsat-5 in Bolivia in 1984 and 1998(Courtesy: Compton J. Tucker and the Landsat Project, NASA Goddard Space Flight Center)

- Influence of image registration on products validation

- Impact of misregistration on legal, economic and sociopolitical (e.g., resource management), etc. 


\section{Image Registration Frameworks}

- Mathematical Framework

- I1(x,y) and I2(x,y): images or image/map

- find the mapping (f,g) which transforms I1 into I2: I2(x,y) $=\mathbf{g}(\mathbf{I} 1(\mathbf{f x}(\mathbf{x}, \mathbf{y}), \mathbf{f y}(\mathbf{x}, \mathbf{y}))$

» $\mathrm{f}$ : spatial mapping

» g: radiometric mapping

- Spatial Transformations "f"

- Translation, Rigid, Affine, Projective, Perspective, Polynomial, ...

- Radiometric Transformations "g” (Resampling)

- Nearest Neighbor, Bilinear, Cubic Convolution, ...

- Algorithmic Framework (Brown, 1992)

1. Feature Extraction

2. Feature Matching

3. Image Resampling 


\section{NASA Goddard Image Registration Group}

- 1994: First results on the utilization of orthogonal Daubechies wavelets for image registration
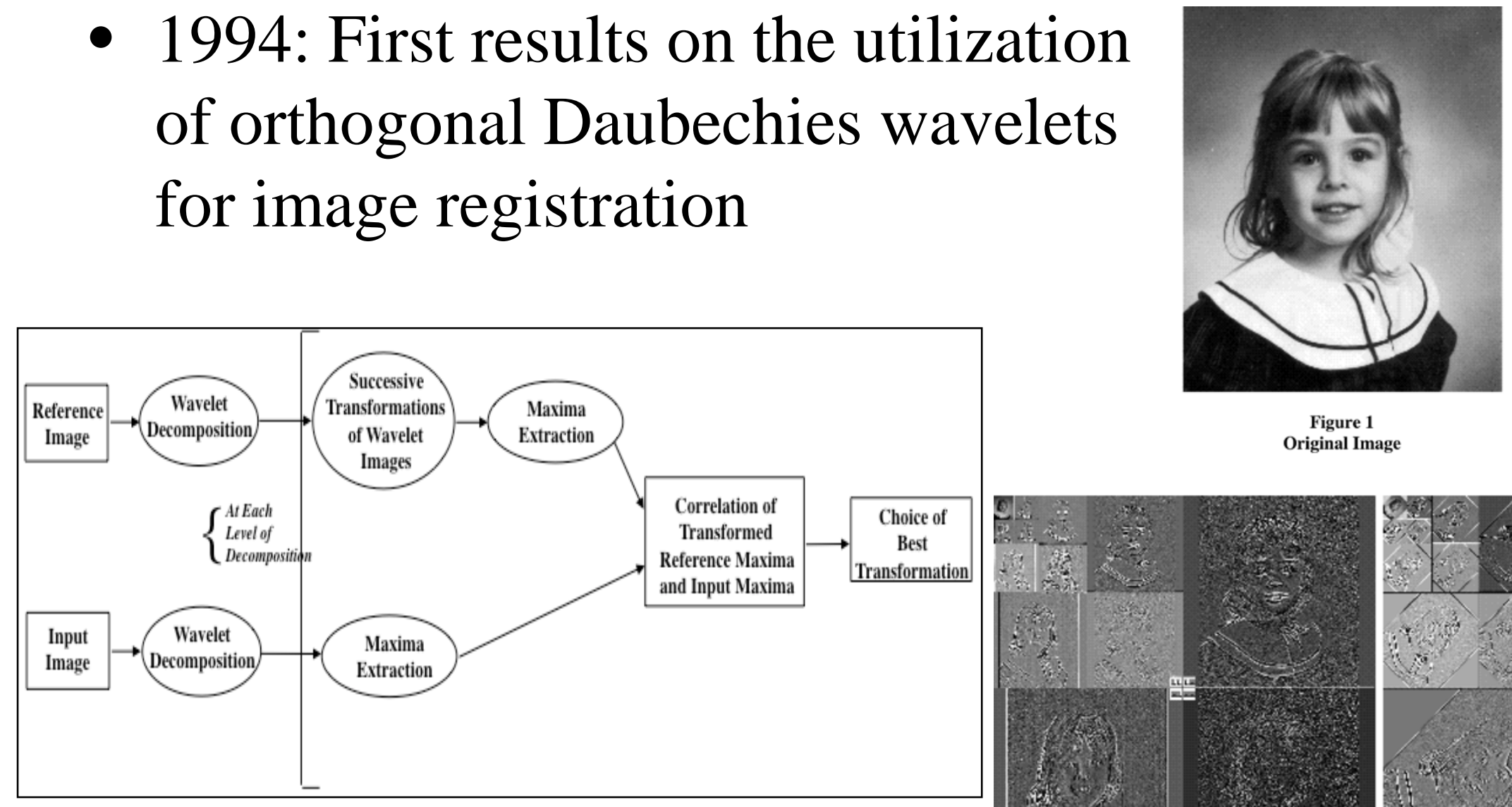

Figure 1 Original Image

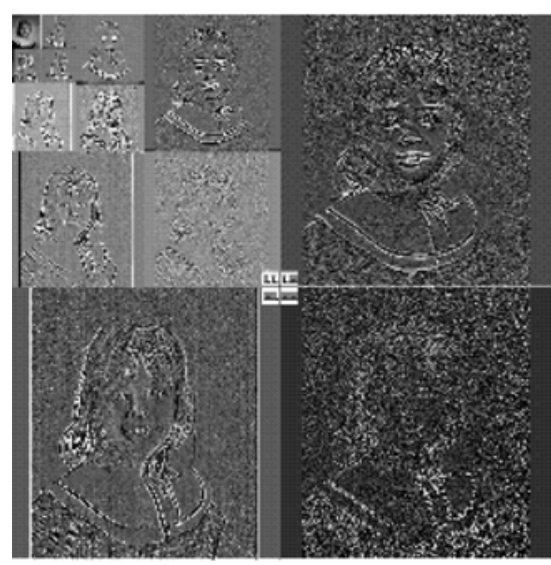

Figure 2

Wavelet Coefficients Corresponding to Figure 1

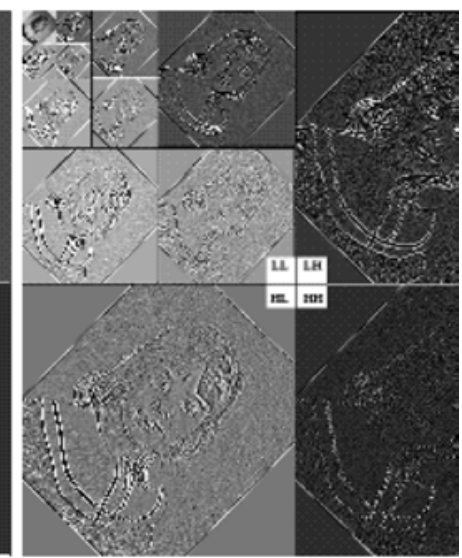

Figure 3

Wavelet Coefficients Correspond to Figure 1 rotated 44 degrees 


\section{NASA Goddard Image Registration Group}

- Study of rotation- and translation-invariant wavelet filters (Spline, Simoncelli)

- Study of different matching strategies and metrics

- Parallel implementations (SIMD/MasPar, Beowulf Cluster, MIMD/Cray-T3E, FPGA-Hybrid)

- Development of image registration framework based on Brown's framework

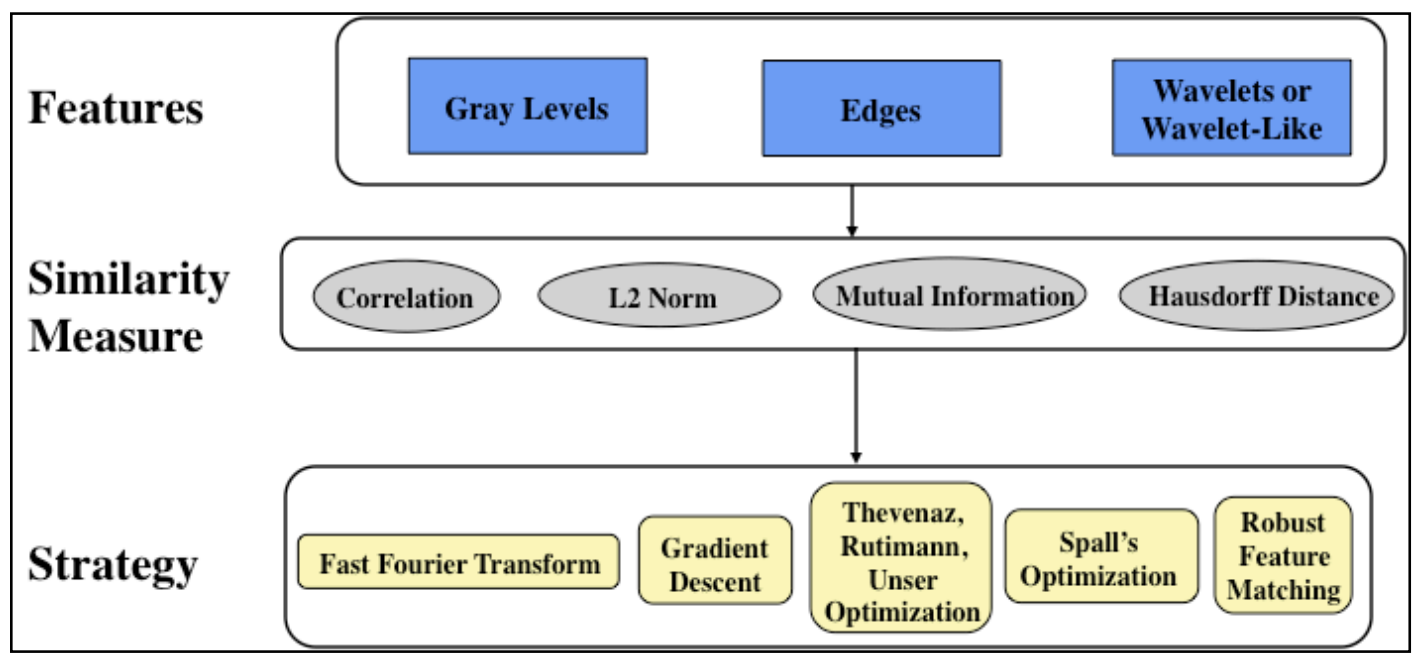




\section{Experiments ... Datasets (1)}

- Synthetic Data Experiments

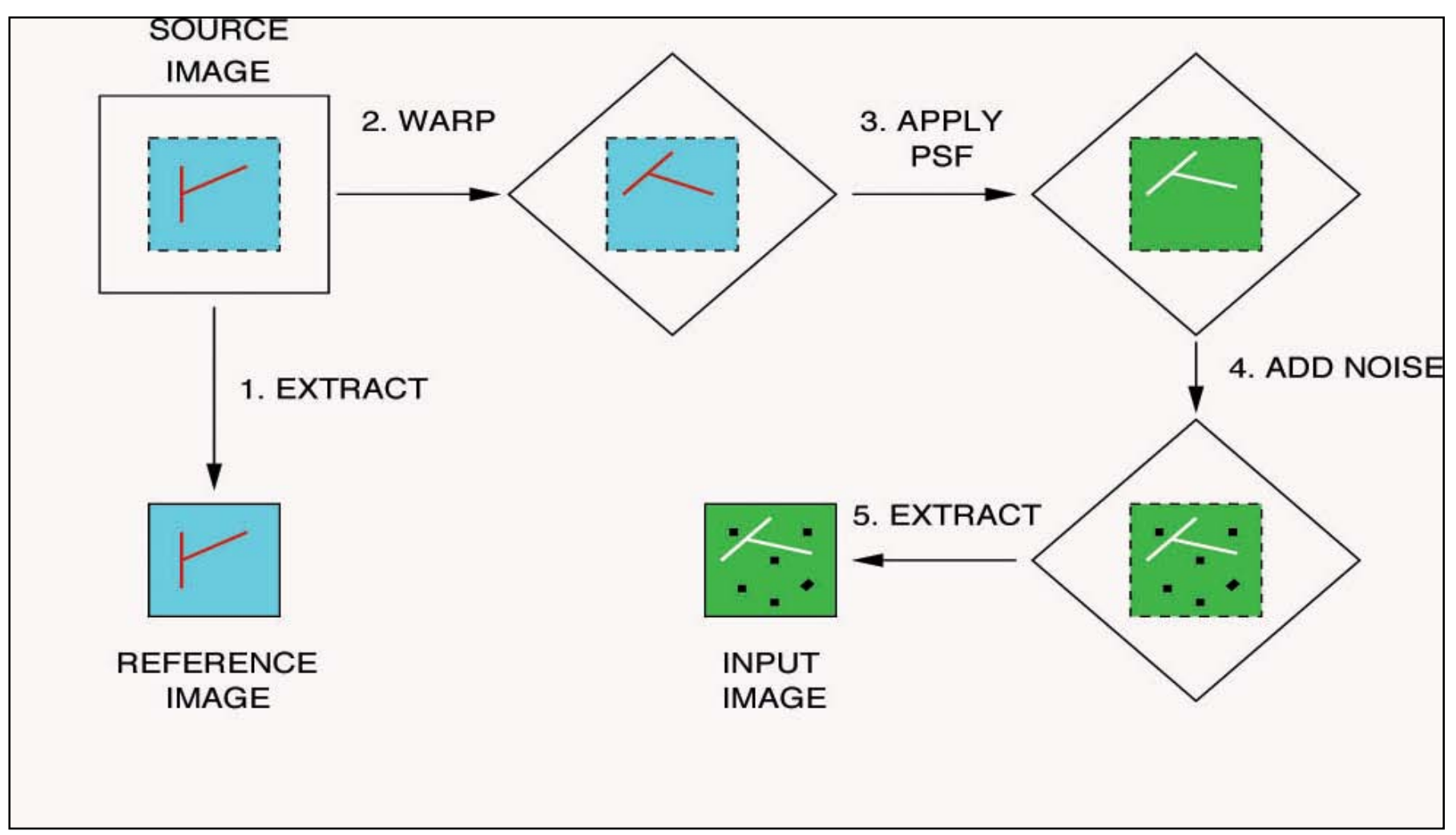




\section{Experiments (1) ... Analysis Samples}

- Various Features; Convergence as a function of noise and radiometric variations

(white areas - regions of convergence with errors less than threshold, e.g. 0.5)
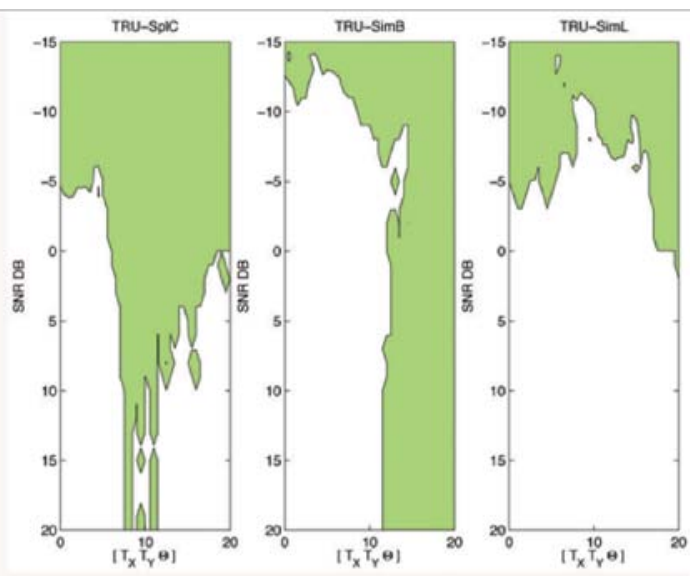

Warping + Noise
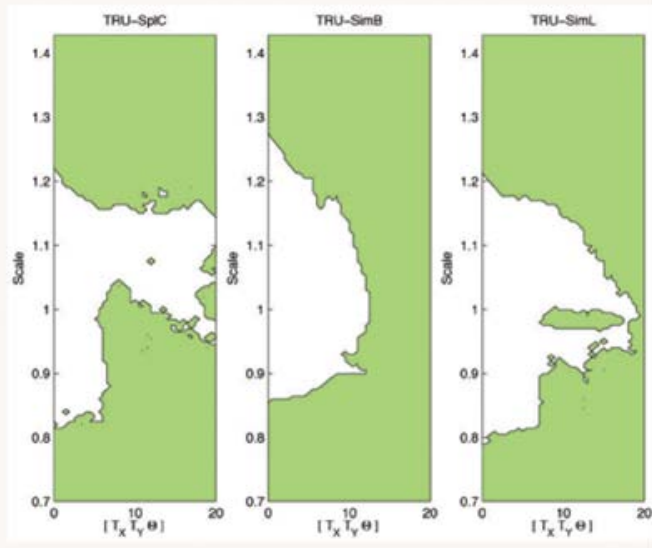

Warping + PSF

- Simoncelli-based methods outperform Spline pyramid-based methods

- Optimization based on Mutual Information does not perfom better than L2-Norm

- Simoncelli-LowPass better than SimoncelliBandPass for Low Noise and Same Radiometry and for Initial Guess Sensitivity
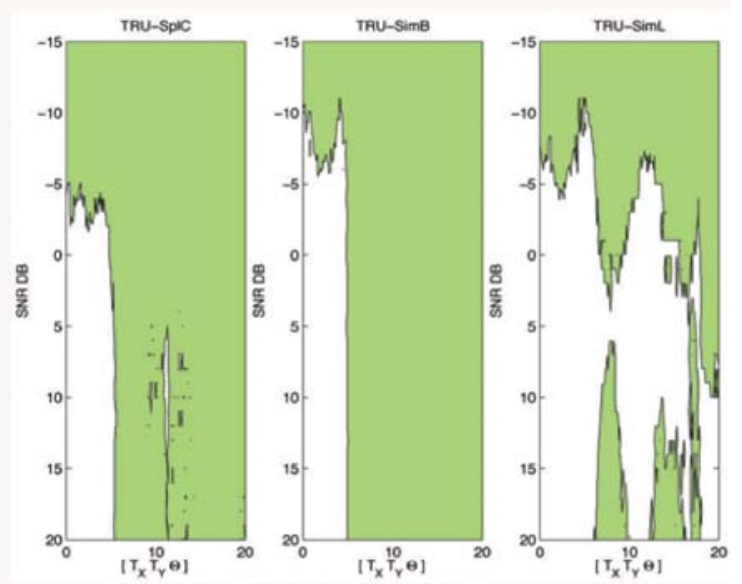

Warping + PSF + Noise 


\section{Experiments ... Datasets (2)}

- Multi-Temporal Data

- Landsat-5 and -7 (chips and corresponding windows)
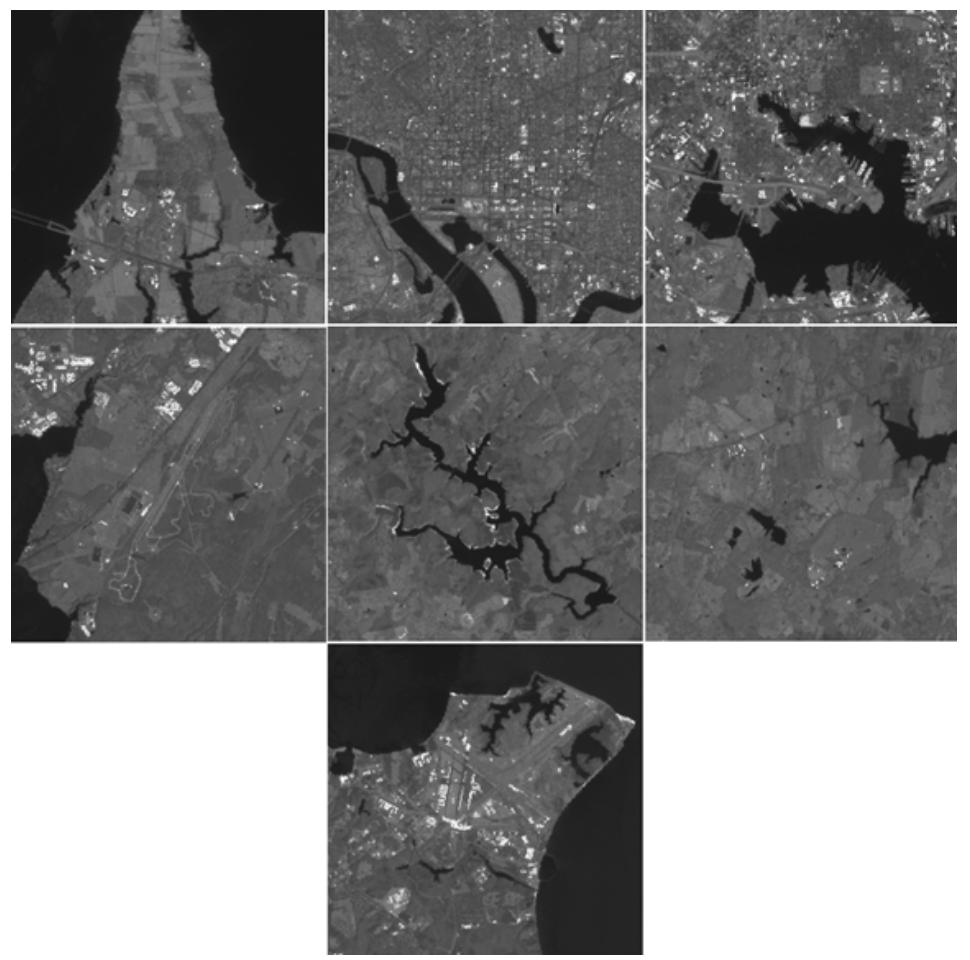

7 Landsat chips

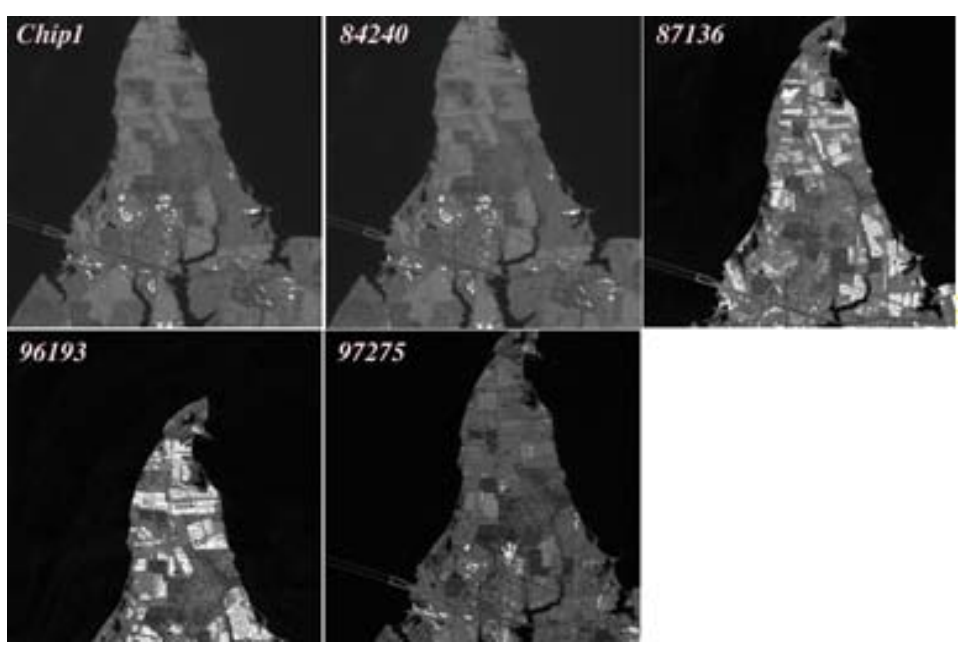

1 Landsat chip and 4 corresponding windows 


\section{Experiments ... Datasets (3)}

- Multi-Sensor Data

- EOS Validation Core Sites

- IKONOS/Landsat-7/MODIS/SeaWiFS

- Red and NIR bands for each sensor

- Spatial resolutions: IKONOS: 4m; ETM+: 30m; MODIS: 500m; SeaWiFS: $1000 \mathrm{~m}$

- 4 different sites:

- Coastal Area: VA, Coast Reserve Area, October 2001

- Agriculture Area: Konza Prairie in State of Kansas, July to August 2001

- Mountainous Area: Cascades Site, September 2000

- Urban Area: USDA Site, Greenbelt, MD, May 2001 


\section{Experiments ... Datasets (3)}

- Multi-Sensor Data

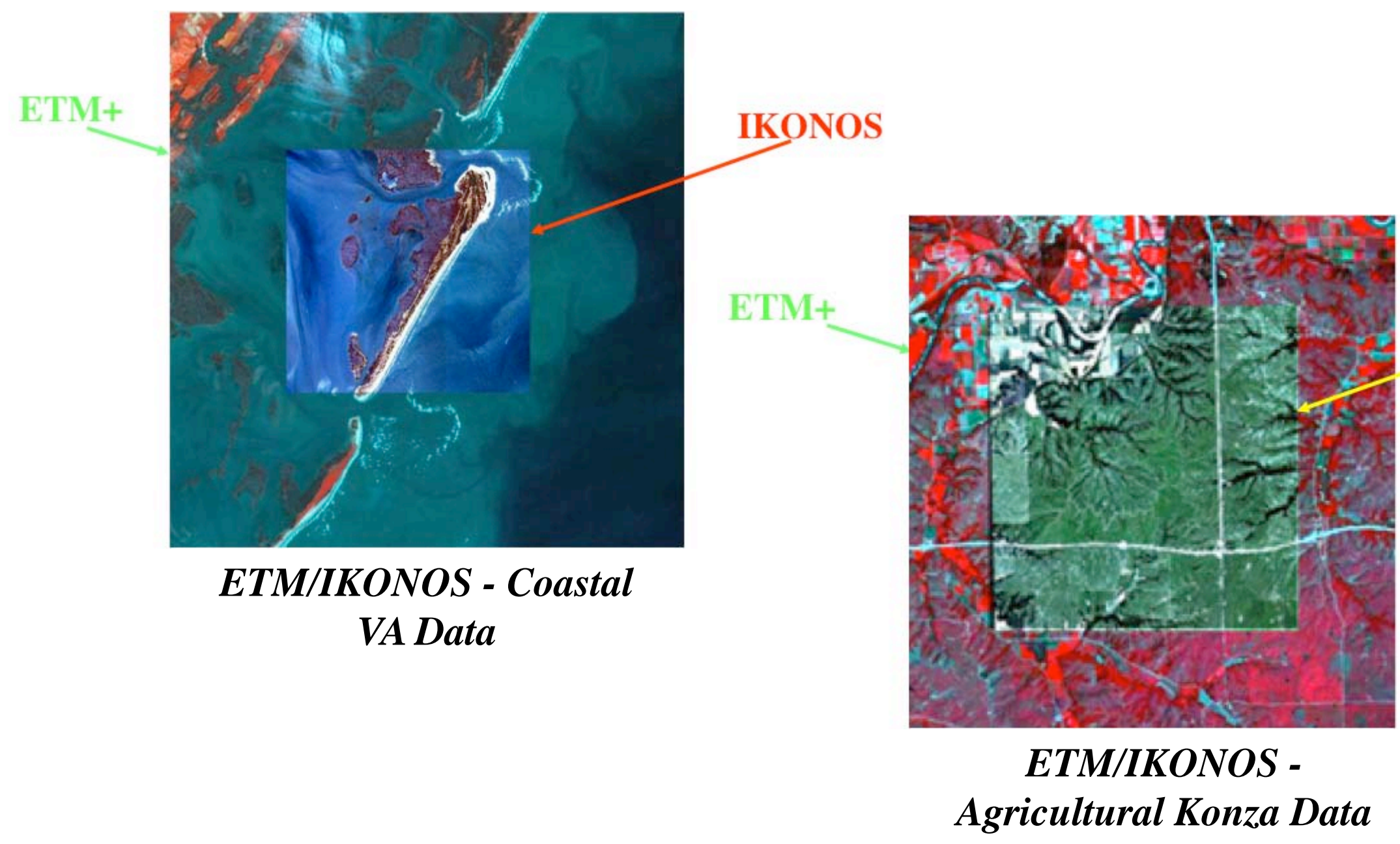




\section{Experiments (2 and 3) ... Analysis Samples}

\begin{tabular}{|c|c|c|c|c|c|c|c|c|c|}
\hline & \multicolumn{3}{|c|}{ RFM REGISTRATION } & \multicolumn{3}{|c|}{$\begin{array}{c}\text { MANUAL GROUND } \\
\text { TRUTH } \\
\end{array}$} & \multicolumn{3}{|c|}{ ABSOLUTE ERROR } \\
\hline Scene & Q & $T_{x}$ & $\mathrm{~T}_{\mathrm{v}}$ & Q & $T_{x}$ & $\mathrm{~T}_{\mathrm{v}}$ & $\mid \mathrm{DQ}$ & $\left|\mathrm{DT}_{\mathrm{x}}\right|$ & $\left|\mathrm{DT}_{\mathrm{v}}\right|$ \\
\hline 840827 & 0.031 & 4.72 & -46.88 & 0.026 & 5.15 & -46.26 & 0.005 & 0.43 & 0.62 \\
\hline 870516 & 0.051 & 8.49 & -45.62 & 0.034 & 8.58 & -45.99 & 0.017 & 0.09 & 0.37 \\
\hline 900812 & 0.019 & 17.97 & -33.36 & 0.029 & 15.86 & -33.51 & 0.010 & 0.11 & 0.15 \\
\hline 960711 & 0.049 & 8.34 & -101.97 & 0.031 & 8.11 & -103.18 & 0.018 & 0.23 & 1.21 \\
\hline
\end{tabular}

Global transformation vs. manual registration (or "ground truth") parameters for 4 Scenes in DC mutitemporal dataset

\begin{tabular}{|c|c|c|c|}
\hline Image Name & \multicolumn{2}{|c|}{ Computed X } & \\
\hline IKONOS red & \multicolumn{2}{|c|}{0} & \\
\hline IKONOS nir & \multicolumn{2}{|c|}{-0.2500} & \\
\hline IKONOS nir & \multicolumn{2}{|c|}{-0.2500} & \\
\hline \multicolumn{4}{|c|}{$\begin{array}{r}\text { Self-Consistency Sti } \\
\text { Informatio }\end{array}$} \\
\hline \multicolumn{2}{|l|}{$\begin{array}{l}\text { SPSA } \\
\end{array}$} & \multicolumn{2}{|c|}{ FCC } \\
\hline SimB & SimL & & \\
\hline$\overline{3}$ & 7 & $\overline{30}$ & \\
\hline 4 & 11 & 30 & \\
\hline 5 & 15 & 30 & \\
\hline 12 & 17 & 30 & \\
\hline 16 & 20 & 30 & \\
\hline 17 & 24 & 30 & \\
\hline 26 & 27 & 30 & \\
\hline 27 & 28 & 30 & \\
\hline 29 & 32 & 30 & \\
\hline 32 & 32 & 30 & \\
\hline 32 & 32 & 30 & \\
\hline
\end{tabular}

\begin{tabular}{|c|c|c|c|c|c|c|c|c|c|c|}
\hline \multirow{2}{*}{\begin{tabular}{|l|} 
\# of CVce \\
Out of 32 \\
\end{tabular}} & \multicolumn{3}{|c|}{ TRU } & \multicolumn{3}{|c|}{ TRUMI } & \multicolumn{3}{|c|}{ SPSA } & \multirow[t]{2}{*}{$\overline{\text { FCC }}$} \\
\hline & SplC & SimB & SimL & SplC & SimB & SimL & SplC & SimB & SimL & \\
\hline 0.0 & 7 & 5 & 12 & 10 & 2 & 14 & 5 & 3 & 7 & 30 \\
\hline 0.1 & 8 & 4 & 14 & 8 & 4 & 12 & 5 & 4 & 11 & 30 \\
\hline 0.2 & 8 & 6 & 16 & 8 & 7 & 15 & 7 & 5 & 15 & 30 \\
\hline 0.3 & 8 & 8 & 16 & 11 & 6 & 19 & 11 & 12 & 17 & 30 \\
\hline 0.4 & 10 & 14 & 21 & 10 & 9 & 17 & 16 & 16 & 20 & 30 \\
\hline 0.5 & 15 & 19 & 25 & 15 & 12 & 21 & 17 & 17 & 24 & 30 \\
\hline 0.6 & 16 & 23 & 27 & 15 & 16 & 25 & 22 & 26 & 27 & 30 \\
\hline 0.7 & 22 & 26 & 28 & 20 & 26 & 29 & 24 & 27 & 28 & 30 \\
\hline 0.8 & 24 & 31 & 31 & 27 & 28 & 30 & 31 & 29 & 32 & 30 \\
\hline 0.9 & 30 & 32 & 31 & 29 & 32 & 31 & 32 & 32 & 32 & 30 \\
\hline 1.0 & 31 & 32 & 31 & 32 & 32 & 31 & 32 & 32 & 32 & 30 \\
\hline
\end{tabular}

Number of cases that converge (out of 32) for the DC dataset, running 4 algorithms and different features with the initial guess varying between the origin (d=0.0) and ground truth (d=1.0)

GOAL: DEFINE A "REGION OF CONVERGENCE” AND A “REGION OF DIVERGENCE” FOR EACH ALGORITHM

$\rightarrow$ RECOMMENDATION FOR UTILIZATION OF ALGORITHMS AND ITS COMPONENTS 


\section{Toolbox for Registration and Analysis (TARA)}
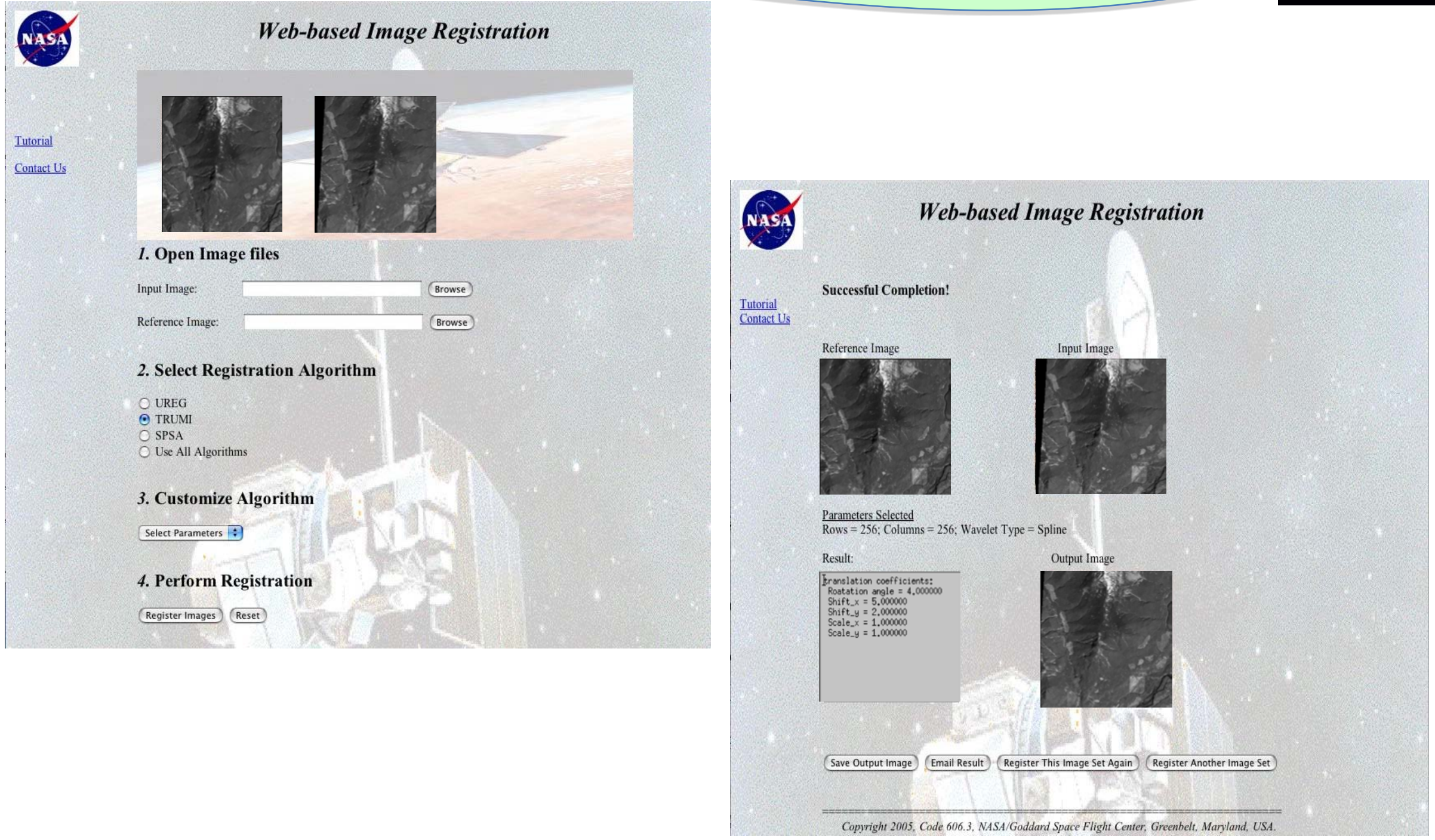


\section{THE BOOK $\ldots$}

- Image Registration for Remote Sensing, ed. J. Le Moigne, N.S. Netanyahu and R.D. Eastman, Cambridge, UK:Cambridge University Press

- Foreword by Jón A. Benediktsson

- Contributors: S. Baillarin/CNES; D.G. Baldwin/Univ. of Colorado; M. Bernard/SPOT Image; A. Bouillon/Institut Géographique National; J.L. Carr/Carr Astronautics; R. Chellappa/UMD; Q-S. Chen/Hickman Cancer Center; A. ColeRhodes/Morgan State Univ.; R.I. Crocker/Univ. of Colorado; R. Davies/Univ. of Auckland; D.J. Diner/NASA JPL; W.J. Emery/Univ. of Colorado; A.A. Goshtasby/Wright State Univ.; V.M. Govindu/Indian Institute of Science; V.M. Jovanovic/NASA JPL; C.S. Kenney/UC Santa Barbara; B.S. Manjunath/UC Santa Barbara; J. Morisette/USGS; D.M. Mount/UMD; M. Nishihama/Raytheon @NASA GSFC; F.S. Patt/SAIC @NASA GSFC; S. Ratanasanya/form. UMD; K. Solanki/UC Santa Barbara; H.S. Stone/form. NEC Research Lab; J. Storey/SGT @USGS; S. Sylvander/CNES; B. Tan/ERT @NASA GSFC; P.K. Varshney/Syracuse Univ.; R.E. Wolfe/NASA GSFC; C. Woodcock/Boston Univ.; M. Xu/Syracuse Univ.; I. Zavorin/form. UMBC@NASA GSFC; M. Zuliani/UC Santa Barbara 


\section{THE BOOK CONTENTS}

- Part I - The Importance of Image Registration for Remote Sensing

- Part II - Similarity Metrics for Image Registration

- Part III - Feature Matching and Strategies for Image Registration

- Part IV-Applications and Operational Systems

- Part V-Conclusion and the Future of Image Registration 
Feature Matching

Feature (Extraction), Similarity Metrics,

Transformations, and Matching Strategies

Nathan S. Netanyahu

Dept. of CS, Bar-Ilan University, Israel, and CfAR/UMIACS, Univ. of Maryland 


\section{Problem Statement}

- Given a reference image, $I_{1}(x, y)$, and a sensed image $I_{2}(x, y)$, find the mapping $\left(T_{p}, g\right)$ which "best" transforms $I_{1}$ into $I_{2}$, i.e.,

$$
I_{2}(x, y)=g\left(I_{1}\left(T_{p}(x, y), T_{p}(x, y)\right)\right),
$$

where $T_{p}$ denotes spatial mapping and $g$ denotes radiometric mapping.

- Spatial transformations:

- Translation, rigid, affine, projective, perspective, polynomial - Radiometric transformations (resampling):

- Nearest neighbor, bilinear, cubic convolution, spline 


\section{Feature (Extraction)}

- Gray levels

- Salient points

- Edge-like, wavelet coefficients (Simoncelli and Freeman '95)

- Corners (Kearny et al. '87, Harris and Stephens '88, Shi and Tomasi '94)

- Lines

- Contours, regions (Govindu et al. '99)

- Scale invariant feature transform (SIFT), Lowe '04 


\section{Similarity Metrics}

- $L_{2}$-norm:

- Minimize the sum of squared errors (SSD) over overlapping subimage

$$
S S D(x, y)=\sum_{m=0}^{M-1} \sum_{n=0}^{N-1}\left[I_{1}(m, n)-I_{2}(m-x, n-y)\right]^{2}
$$
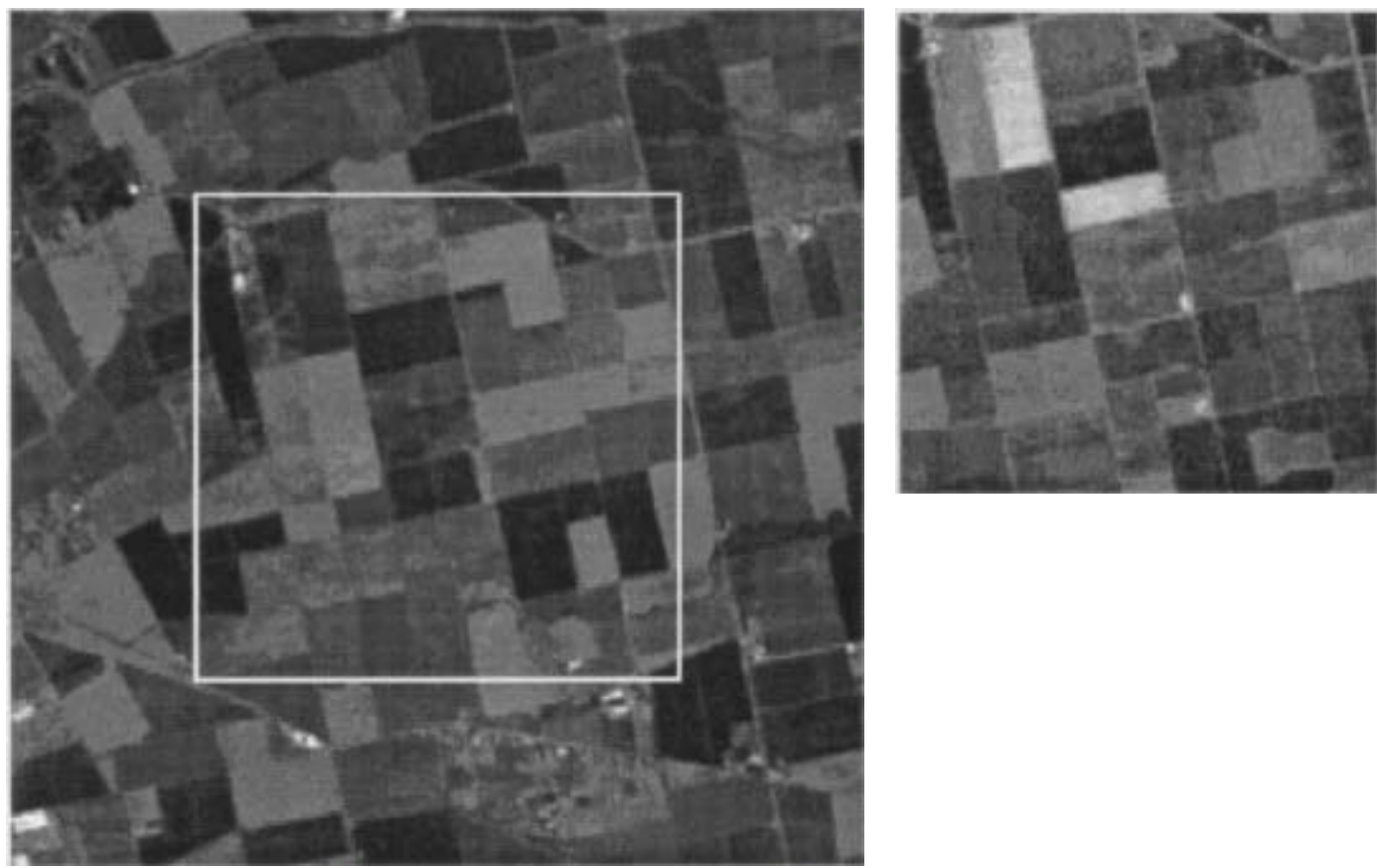


\section{Similarity Metrics (cont’d)}

- Cross-correlation

- Maximize cross-correlation over image overlap

$$
I_{1}(x, y) \circ I_{2}(x, y)=\sum_{m=0}^{M-1} \sum_{n=0}^{N-1} I_{1}(m, n) I_{2}(x+m, y+n)
$$

- Normalized cross-correlation (NCC)

- Maximize normalized cross-correlation

$$
N C C_{I_{1}, I_{2}}(x, y)=\frac{\sum_{m=0}^{M-1} \sum_{n=0}^{N-1}\left[I_{1}(m, n)-\bar{I}_{1}\right]\left[I_{2}(x+m, y+n)-\bar{I}_{2}\right]}{\sqrt{\sum_{m=0}^{M-1} \sum_{n=0}^{N-1}\left[I_{1}(m, n)-\bar{I}_{1}\right]^{2} \cdot \sum_{m=0}^{M-1} \sum_{n=0}^{N-1}\left[I_{2}(x+m, y+n)-\bar{I}_{2}\right]^{2}}}
$$




\section{Similarity Metrics (cont’d)}

- Mutual information (MI):

Maximizes the degree of statistical dependence between the images

$$
M I\left(I_{1}, I_{2}\right)=\sum_{g_{1}} \sum_{g_{2}} p_{I_{1}, I_{2}}\left(g_{1}, g_{2}\right) \cdot \log \left(\frac{p_{I_{1}, I_{2}}\left(g_{1}, g_{2}\right)}{p_{I_{1}}\left(g_{1}\right) \cdot p_{I_{2}}\left(g_{2}\right)}\right),
$$

or using histograms, maximizes

$$
M I\left(I_{1}, I_{2}\right)=\frac{1}{M} \sum_{g_{1}} \sum_{g_{2}} h_{I_{1}, I_{2}}\left(g_{1}, g_{2}\right) \cdot \log \left(\frac{M h_{I_{1}, I_{2}}\left(g_{1}, g_{2}\right)}{h_{I_{1}}\left(g_{1}\right) \cdot h_{I_{2}}\left(g_{2}\right)}\right)
$$

where $M$ is the sum of all histogram entries, i.e., number of pixels (in overlapping subimage) 


\section{Similarity Metrics (cont’d)}
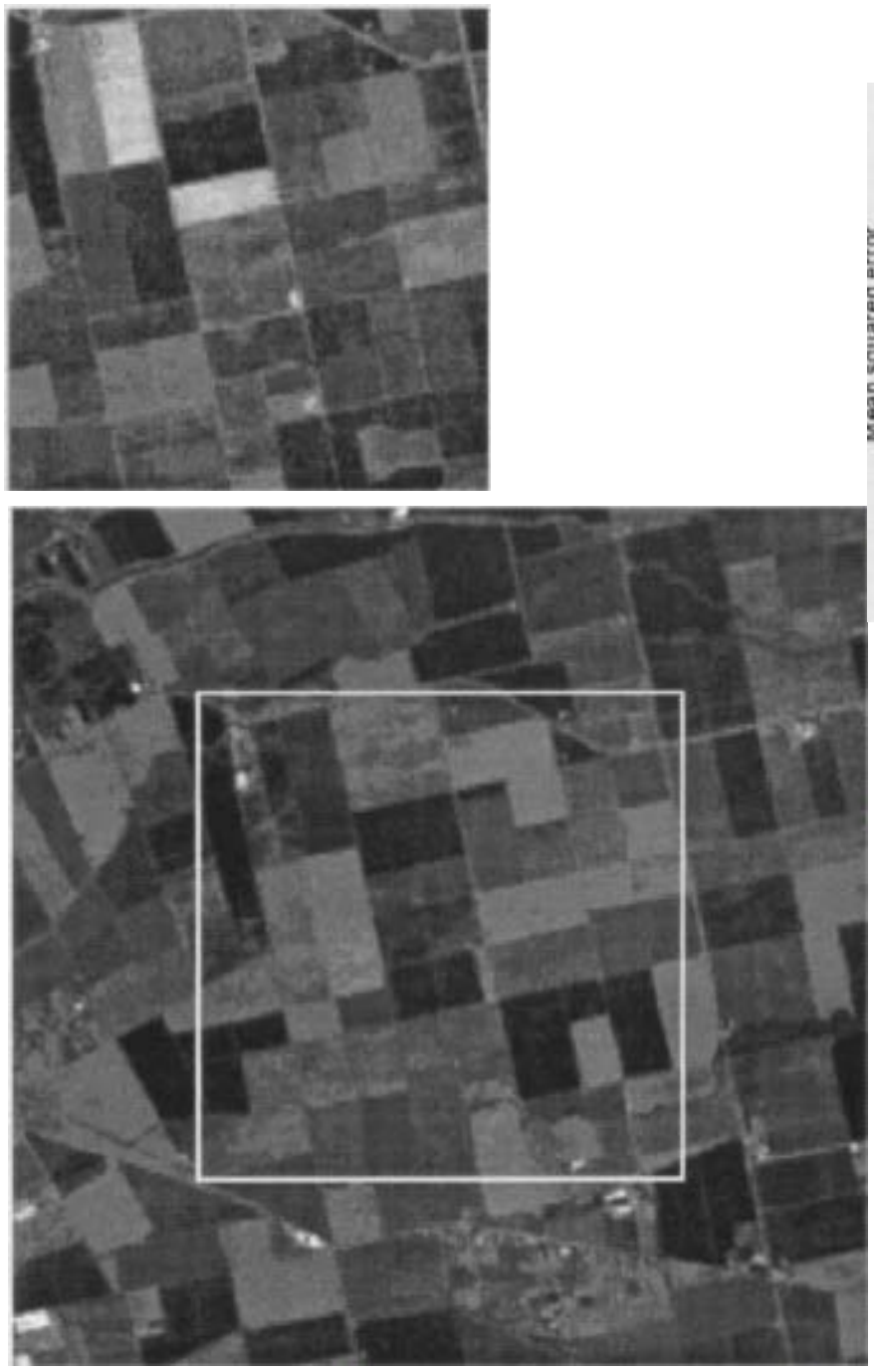

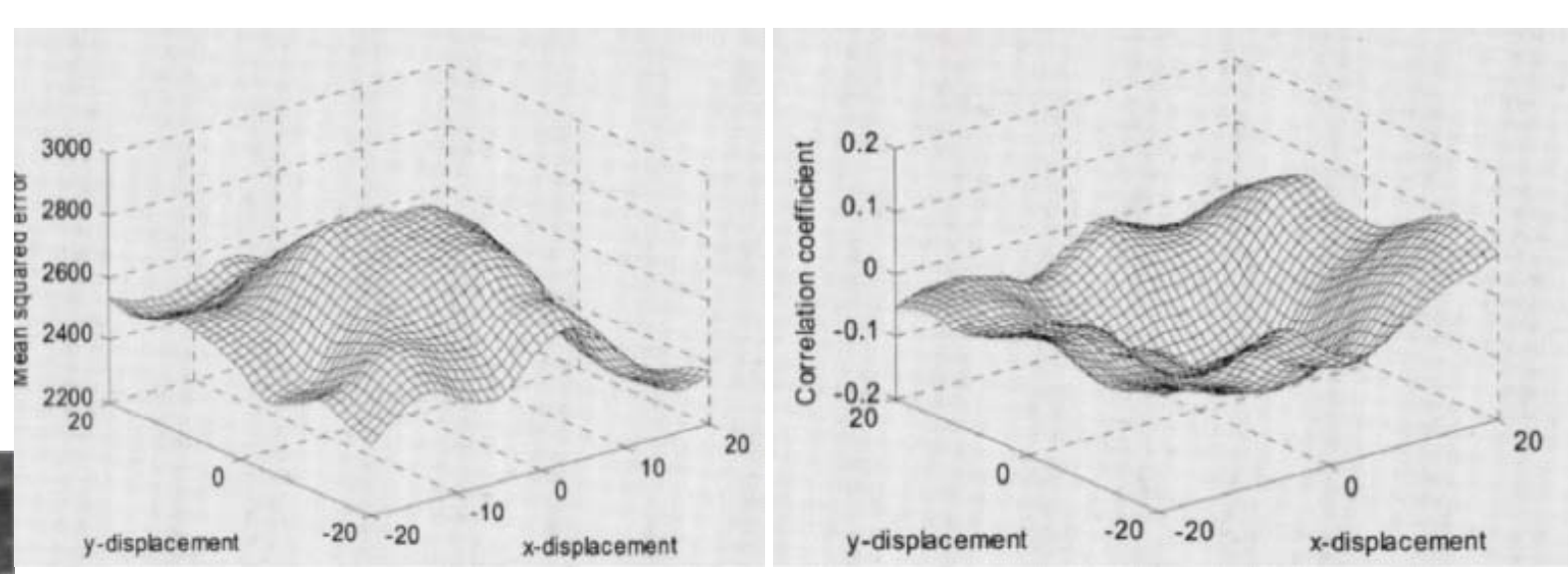

MI vs. $L_{2}$-norm and NCC applied to Landsat-5 images (source: H. Chen et al. '03) 


\section{Similarity Metrics (cont’d)}

- Partial Hausdorff distance (PHD):

$$
H_{K}\left(I_{1}, I_{2}\right)=K_{p_{1} \in I_{1}}^{\text {th }} \min _{p_{2} \in I_{2}} \operatorname{dist}\left(p_{1}, p_{2}\right),
$$

where $1 \leq K \leq\left|I_{1}\right|$ (Huttenlocher et al. '93, Mount et al. '99)

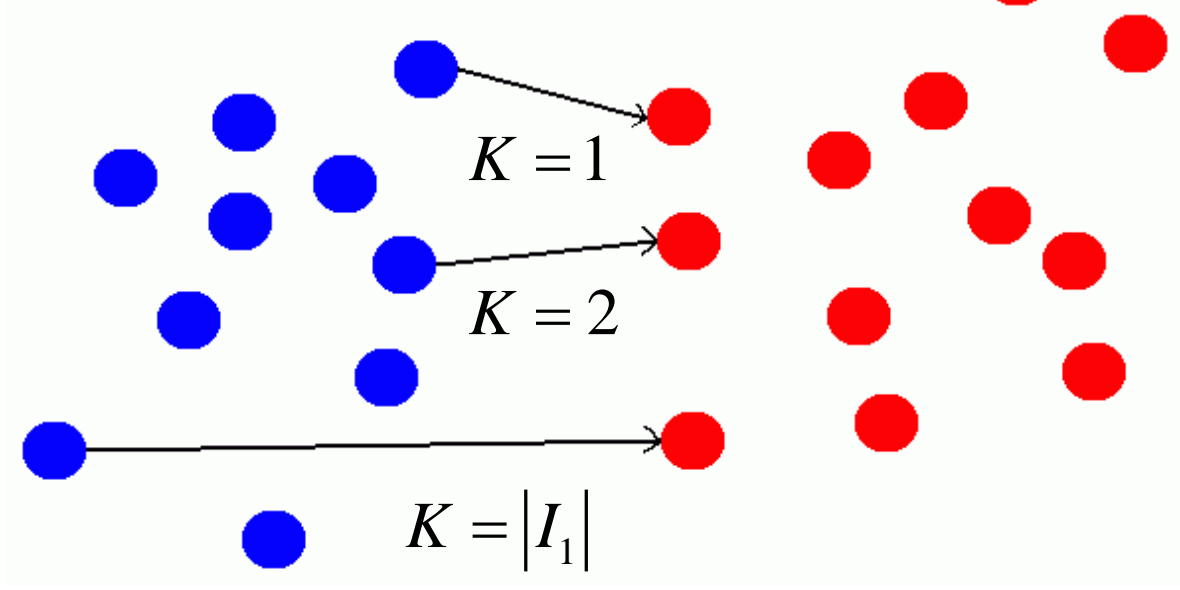




\section{Similarity Metrics (cont'd)}

- Discrete Gaussian mismatch (DGM):

$$
w_{\sigma}(a)=\exp \left(-\frac{\operatorname{dist}\left(a, I_{2}\right)^{2}}{2 \sigma^{2}}\right)
$$

where $w_{\sigma}(a)$ denotes the weight of point $a$, and

$$
\operatorname{DGM}_{\sigma}\left(I_{1}, I_{2}\right)=1-\frac{\sum_{a \in I_{1}} w_{\sigma}(a)}{\left|I_{1}\right|}
$$

is similarity measure ranging between 0 and 1 (Mount et al., Ch. 8) 


\section{Transformation Functions}

- Translation-only, rigid

- Rotation, scale, and translation (RST)

- Affine (6 degrees of freedom)

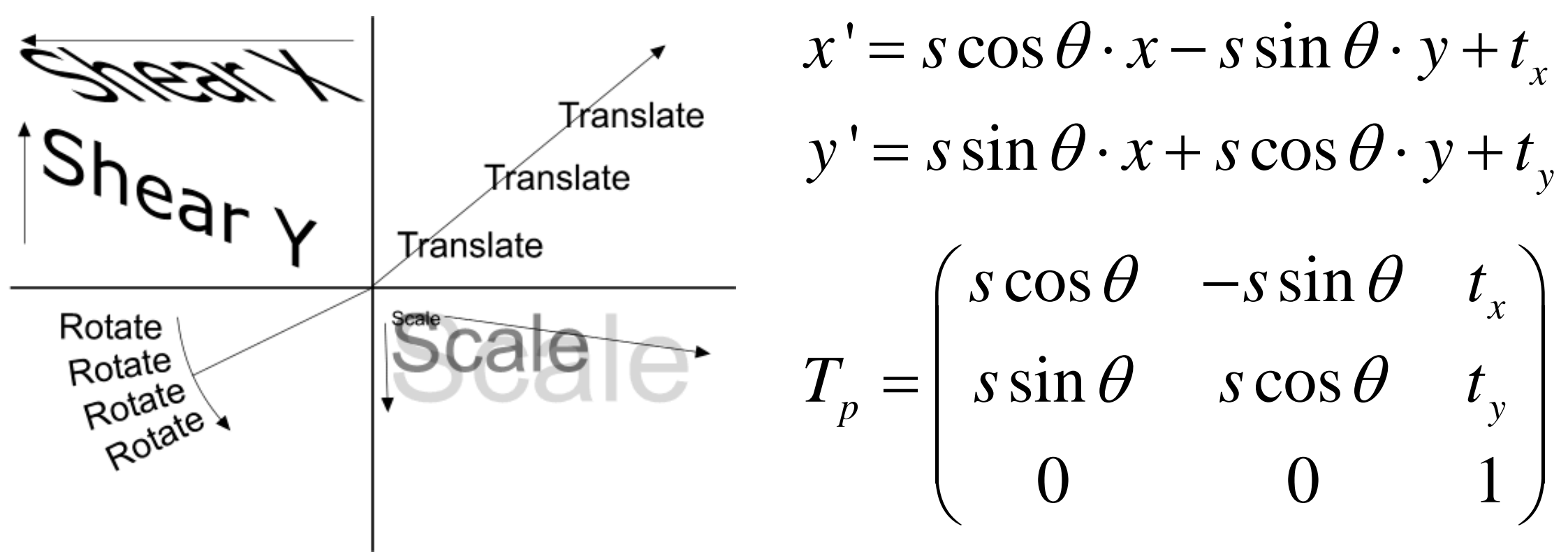

- Projective/homography (e.g., for perspective effects in image mosaicing; Govindu and Chellappa, Ch. 10); 8 parameters 


\section{Transformation Functions (cont’d)}

- Weighted linear transformation (Goshtasby, Ch. 7); adaptive transformation, continuous and smooth, applied to multiview images with local geometric differences, and maps an entire image to another

- Interpolating surface is a weighted sum of planar patches, each of which passes through a control point and provides a desired gradient, i.e.,

$$
f(x, y)=\frac{\sum_{i=1}^{n} R_{i}(x, y) L_{i}(x, y)}{\sum_{i=1}^{n} R_{i}(x, y)}
$$

for monotonically decreasing weight $R_{i}(x, y)=\left[\left(x-x_{i}\right)^{2}+\left(y-y_{i}\right)^{2}\right]^{-1 / 2}$

and

$$
L_{i}(x, y)=a_{i}\left(x-x_{i}\right)+b_{i}\left(y-y_{i}\right)+F_{i}
$$




\section{Transformation Functions (cont'd)}

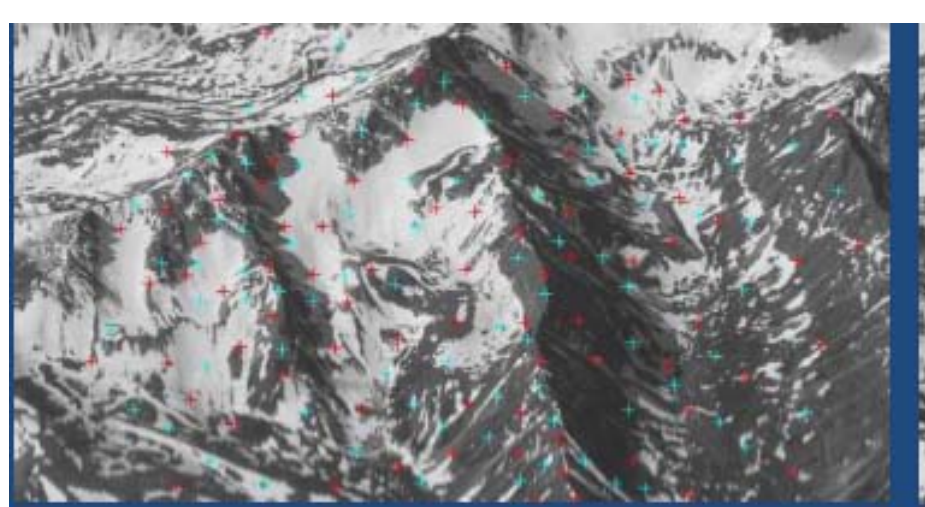

Reference

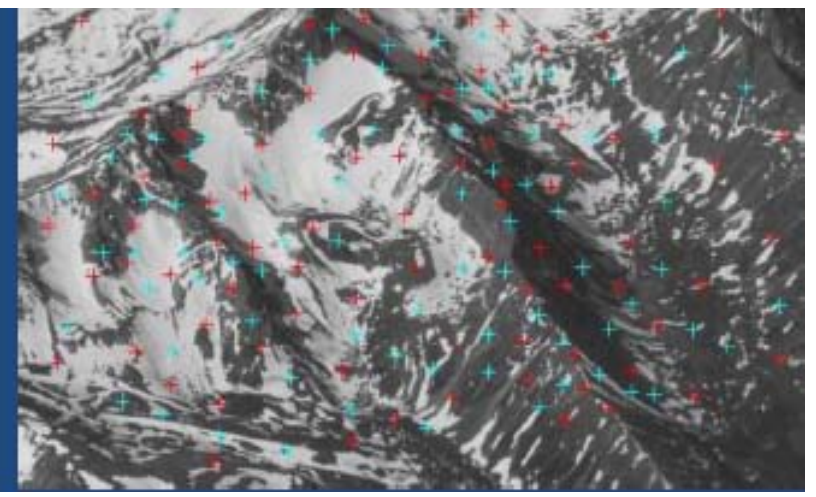

Sensed

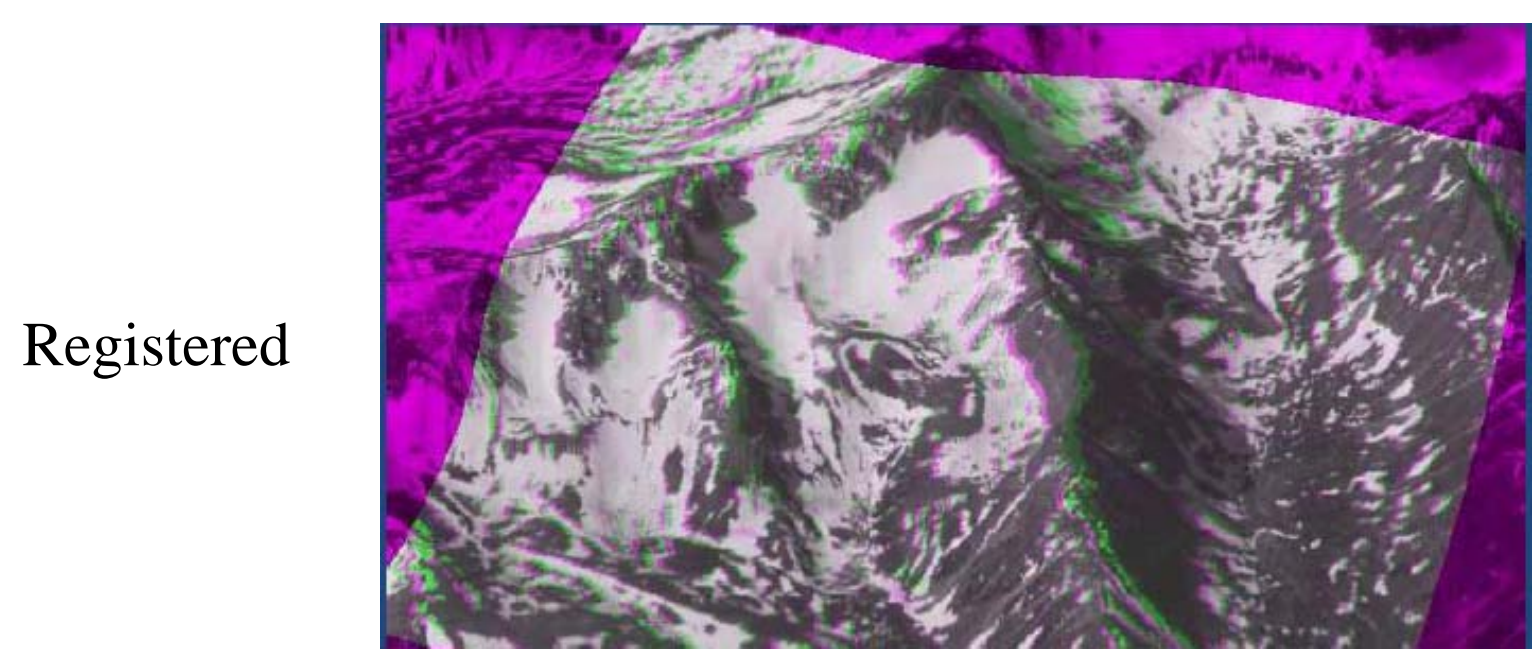

Source: Goshtasby, IR Tutorial, CVPR '11 


\section{IR Components (Revisited)}

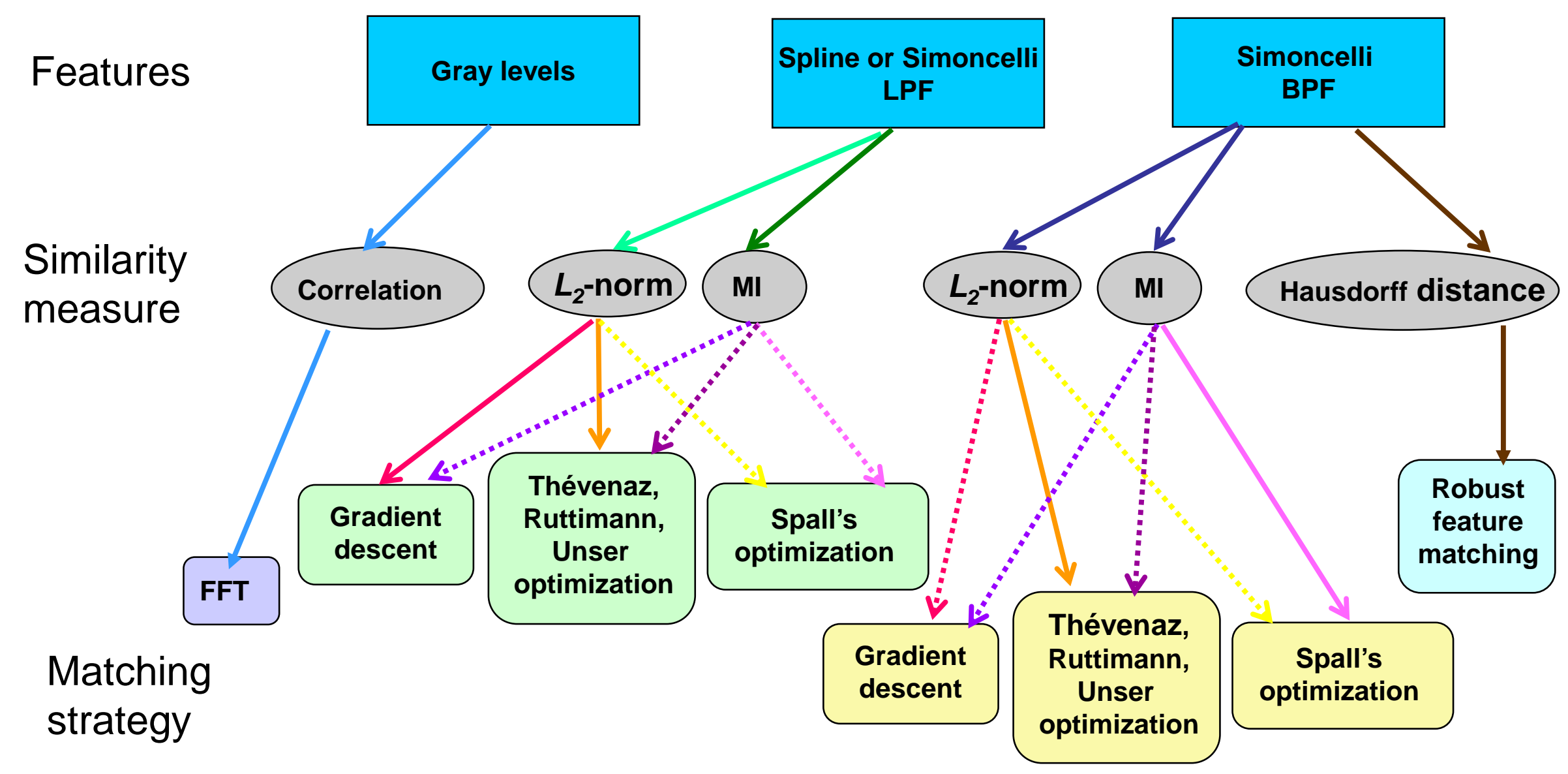




\section{Matching Strategies}

- Exhaustive search (exponential in dimensionality of space)

- Fast Fourier transform (FFT)

- Numerical optimization (e.g., steepest gradient descent wrt SSD, NCC, and MI (Thévenaz, Ruttimann, and Unser (TRU) '98; Spall '92))

- Robust transformation estimate (e.g., RANSAC, LMS) if (most) correspondences are known (via SIFT-like)

- “Correspondenceless", e.g., correlation of descriptor distribution/feature consensus (Govindu et al. '99)

- Robust feature matching (RFM), e.g., efficient subdivision and pruning of transformation space; Huttenlocher et al. '93, Mount et al. '99, Netanyahu et al. '04 


\section{Matching Strategies (cont'd)}

\section{- Frequency domain-based approach (Stone, Ch. 4)}

- Efficient computation of correlation as inverse of $F_{1}^{*}(u, v) F_{2}(u, v)$

- Practical implementation (extension to NCC, masking invalid pixels, optimized computation)

- Finding (small) rotational and scale differences (by matching chips)

- Subpixel registration for translation-only using phase estimate (also in case of image aliasing)

- Rotation and scale estimate by casting to log-polar coordinates 


\section{Matching Strategies (cont'd)}

\section{- Matched filtering (Q. Chen, Ch. 5)}

- Maximize SNR (using theory of linear systems)

- Apply phase-only and symmetric phase-only matched filters for translation-only IR

$$
\text { Phase product }=\frac{F_{1}^{*}(u, v)}{\left|F_{1}(u, v)\right|} \frac{F_{2}(u, v)}{\left|F_{2}(u, v)\right|}=e^{-j\left(u t_{x}+v t_{y}\right)}
$$

- Apply Fourier-Mellin transform for rotation and scale changes; transform represents these parameters as translational shifts in log-polar coordinates of magnitude of Fourier spectrum, i.e., first estimate rotation and scale, followed by translation estimate 


\section{Matching Strategies (cont’d)}

Rotation and scale estimate
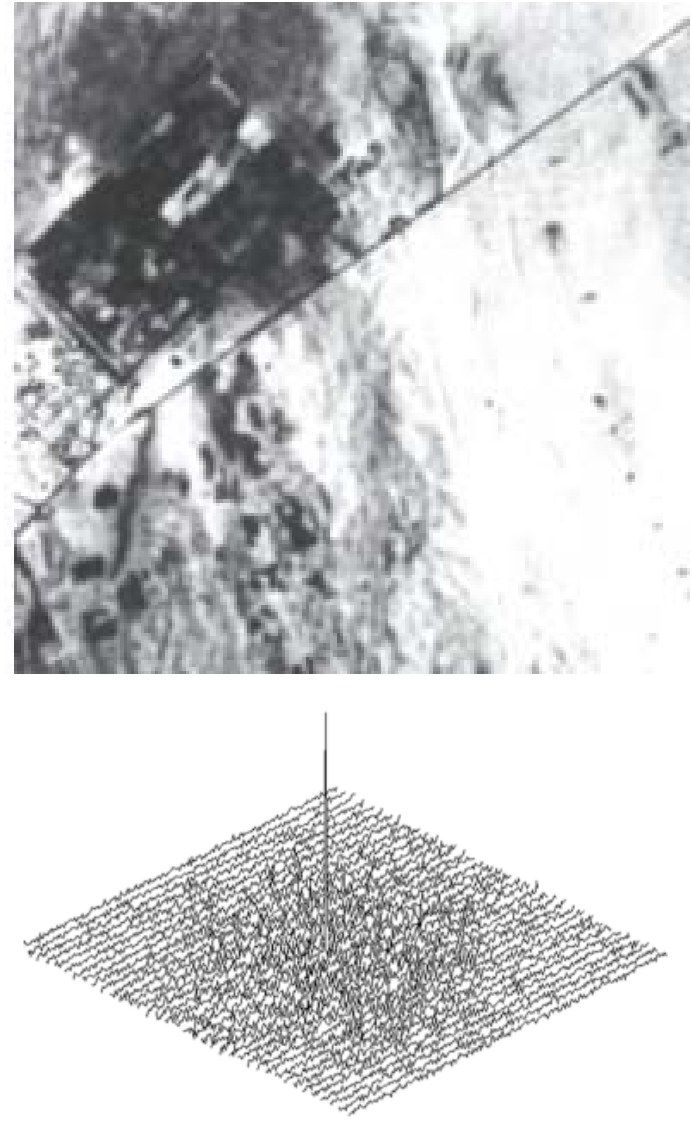

Translation estimate

Pair of SPOT images and their registration, using symmetric phase-only matched filters on their Fourier-Mellin transforms 


\section{Matching Strategies (cont'd)}

- Numerical optimization (Cole-Rhodes and Varshney, Ch. 6; Cole-Rhodes and Eastman, Ch. 12)

- Powel's, Brent's (1-D), simplex, etc.

- Steepest descent/ascent variants

- Standard

$$
\begin{aligned}
& \mathbf{p}_{k+1}=\mathbf{p}_{k}-\lambda_{k} \mathbf{g}_{k} \\
& \mathbf{p}_{k+1}=\mathbf{p}_{k}-\lambda_{k} \mathbf{H}_{k}^{-1} \mathbf{g}_{k} \\
& \mathbf{p}_{k+1}=\mathbf{p}_{k}-\left(\mathbf{H}_{k}+\lambda_{k} \operatorname{diag}\left[\mathbf{H}_{k}\right]\right)^{-1} \mathbf{g}_{k}
\end{aligned}
$$

- Newton-Raphson

- Levenberg-Marquardt

- Apply to various similarity metrics, e.g., SSD (Eastman and Le Moigne '01), MI, etc.

» Explicit computation of gradient (and Jacobian/Hessian), e.g., Thévenaz and Unser '00

» Stochastic approx. (Spall '92); Cole-Rhodes et al. '03; Cole-Rhodes and Varshney, Ch. 6 


\section{Matching Strategies (cont'd)}
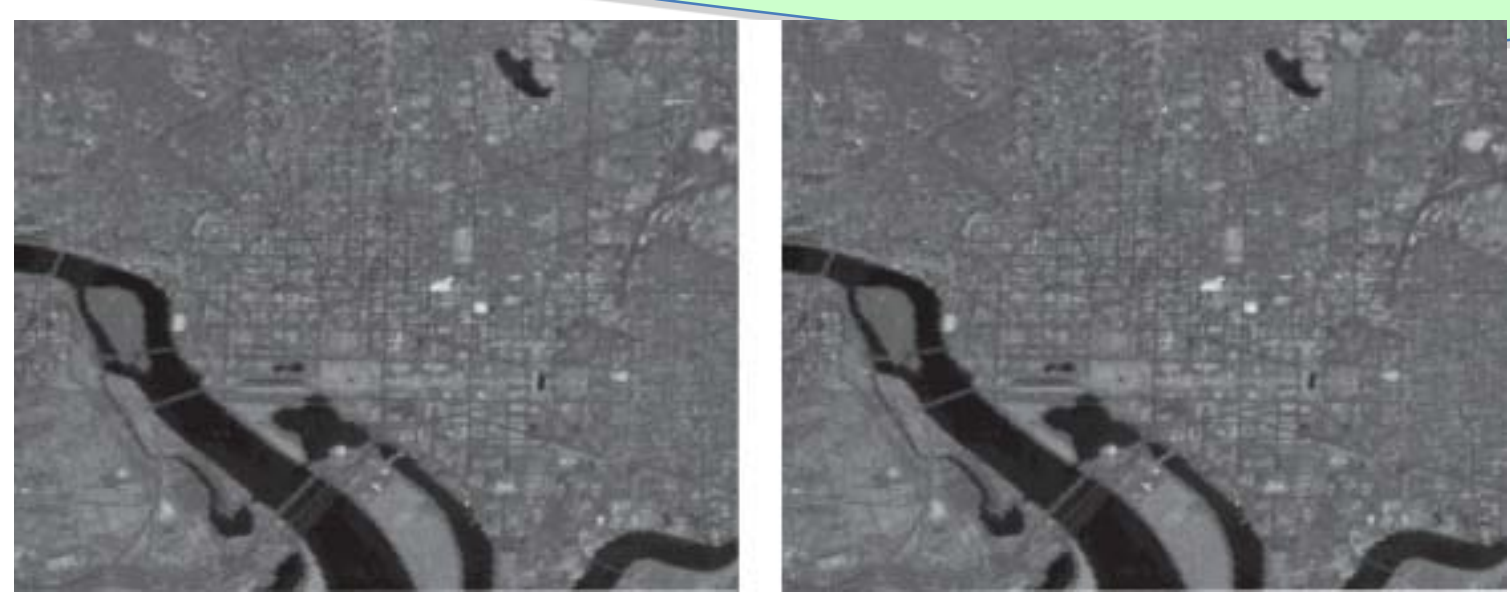

Pair of Landsat images over DC
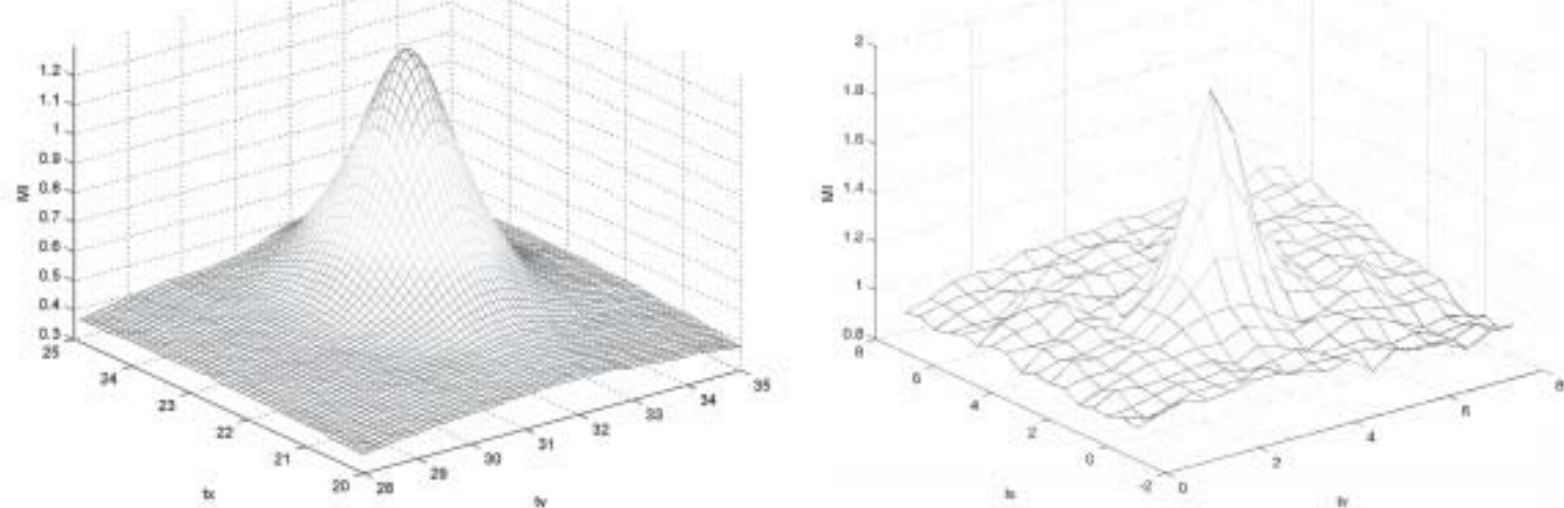

MI surfaces of above (level 1 and 4) images, using B-spline interpolation (Cole-Rhodes and Varshney, Ch. 6) 


\section{Matching Strategy (cont'd)}

- Alignment via local geometric distributions (Govindu and Chellappa, Ch. 10)
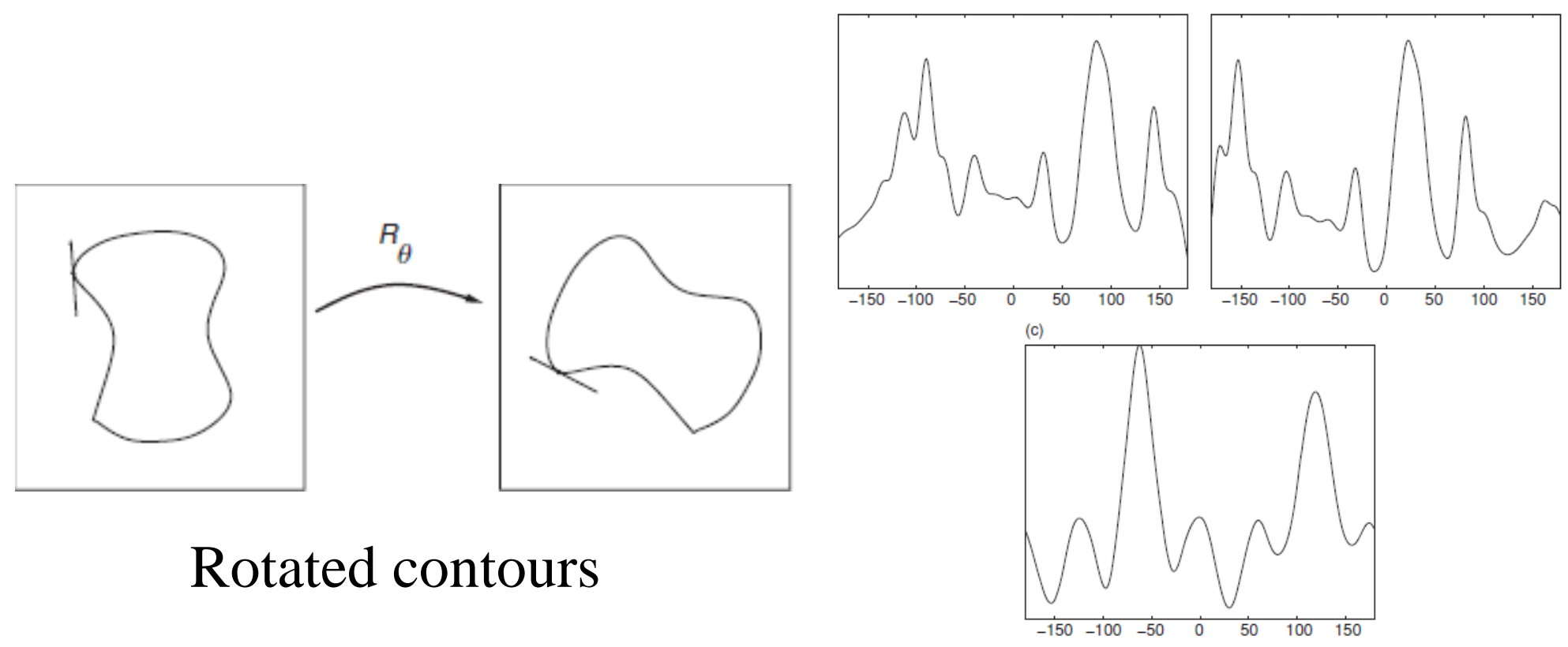

Rotated contours

Slope angle distributions and their correlation 


\section{Matching Strategy (cont'd)}

- Robust feature matching (RFM) (Mount et al., Ch. 8)

- Space of affine transformations: 6-D space

- Subdivide: Quadtree or kd-tree. Each cell $T$ represents a set of transformations; $T$ is active if it may contain $t_{\mathrm{opt}} ; \mathrm{o} / \mathrm{w}$, it is killed

- Uncertainty regions (UR's): Rectangular approximation to the possible images $\tau(a)$ for all $\tau \in T, a \in I_{1}$

- Bounds: Compute upper bound (on optimum similarity) by sampling a transformation and lower bound by computing nearest neighbors to each UR

- Prune: If lower bound exceeds best upper bound, then kill the cell; o/w, split it 


\section{Matching Strategy (cont'd)}
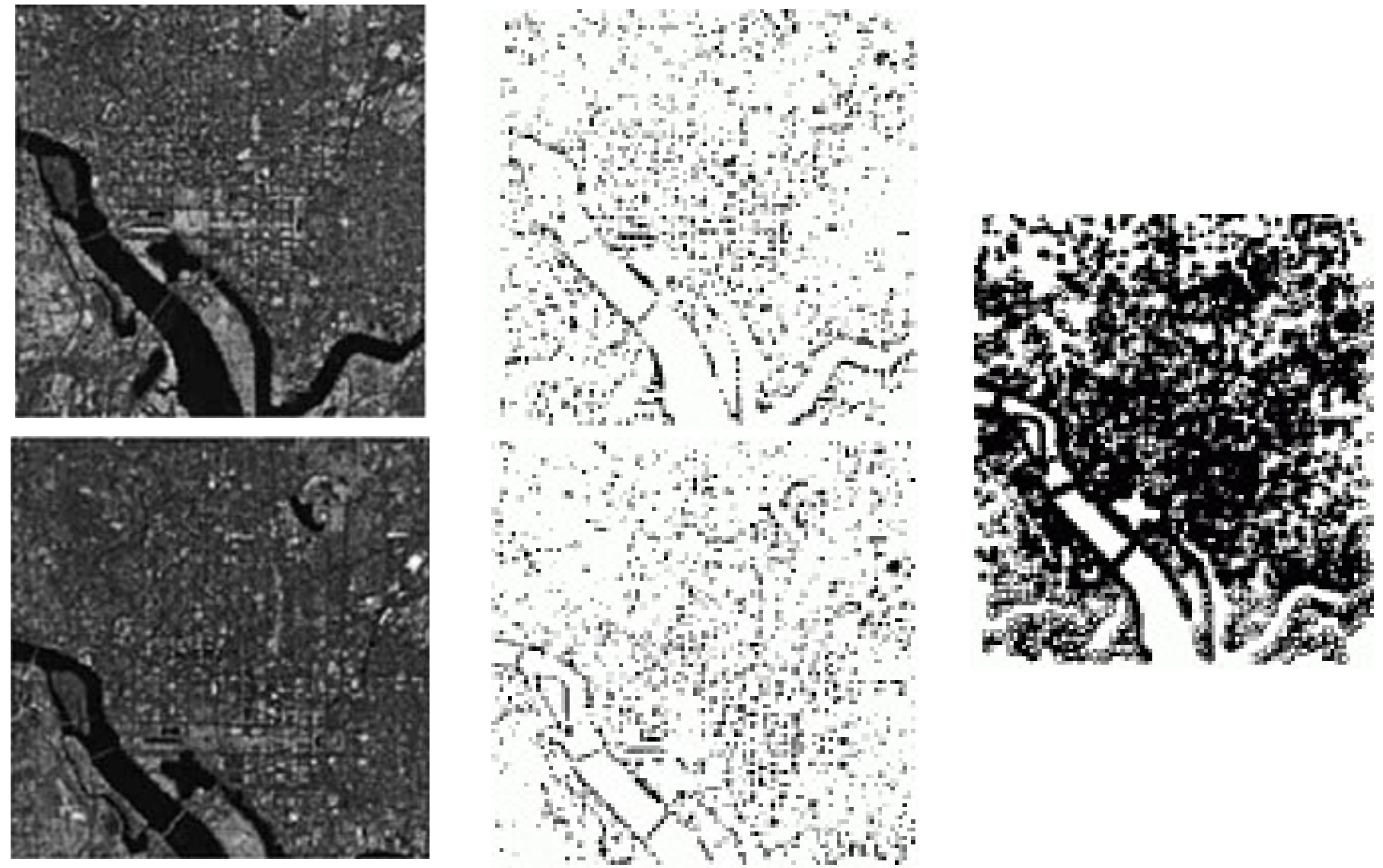

RFM-based registration of Landsat images over DC using wavelet features and PHD similarity measure (Netanyahu et al. '04) 


\section{Matching Strategy (cont'd)}

- Computational efficiency

- "Culling” feature points via, e.g., condition theory (Kenney et al. '03, Ch. 9)

- Efficient numerical or discrete algorithmic procedures

- Hierarchical pyramid-like (wavelet) decomposition

- Use landmark chip database (instead of a large scene) or alternatively, extract automatically corresponding regions of interest using mathematical morphology (Plaza et al. '05, '07) 


\section{Miscellaneous}

- Use Cramér-Rao bounds as performance benchmark for performance evaluation of image registration (Xu and Varshney, Ch. 13) 
From Theory to Practice Operational Requirements

Roger D. Eastman

Loyola University, Baltimore,

Maryland 


\section{Why isn't this problem solved by now?}

- A wealth of approaches!

- SIFT, ASIFT, BSIFT, SIFT/NCC, SIFT/FLOUR

- Beat the problem to death with terminology

- "Assume we have a Banach space ..."

- Many smart people wielding heavy mathematical weapons against a relatively fixed problem- why hasn't the problem yielded? Why no gold standard algorithm? 


\section{But it is solved ... ask LANDSAT}

Operational Satellite Teams solve it every day

-GOES -Carr, Chapter 15

-MISR - Jovanovic et al, Chapter 16

-AVHRR - Emery et al, Chapter 17

-Landsat, Storey, Chapter 18

-SPOT, Ballarin, Chapter 19

-VEGETATION, Sylvander, Chapter 20

-MODIS, Wolfe et al, Chapter 21

-SeaWiFS, Patt, Chapter 22 


\section{And it's often solved the old-fashioned way (2008)}

Normalized Gross'

\begin{tabular}{|l|l|l|l|l|}
\hline Instrument & Satellite & Resolution & Similarity & Subpixel \\
\hline ASTER & Terra & $15 \mathrm{~m}-90 \mathrm{~m}$ & NCC w/ DEM & Fit to surface \\
\hline GOES & GOES I-M & $1 \mathrm{~km}-8 \mathrm{~km}$ & $\begin{array}{l}\text { NCC } \text { w/ vector } \\
\text { coastlines }\end{array}$ & $\begin{array}{l}\text { Bi-section } \\
\text { search }\end{array}$ \\
\hline MISR & Terra & $275 \mathrm{~m}$ & NCC w/ DEM & Least squares \\
\hline MODIS & Terra & $250 \mathrm{~m}-1 \mathrm{~km}$ & NCC w/ DEM & Fixed grid \\
\hline HRS & SPOT & $2.5 \mathrm{~m}$ & NCC w/ DEM & Not described \\
\hline ETM+ & Landsat-7 & $15 \mathrm{~m}-60 \mathrm{~m}$ & $\begin{array}{l}\text { NCC to arid } \\
\text { region CPs }\end{array}$ & Fit to surface \\
\hline VEGETATION & SPOT & $1 \mathrm{~km}$ & NCC w/ DEM & Not described \\
\hline
\end{tabular}




\section{Example: Landsat ETM+}

- Geodetic accuracy

- Database of GCPs derived from USGS data

- Normalized correlation

- Updates navigation models

- Results: RMSE 54m

- Band-to-band registration

- Selected tie-points in high-freq. arid regions

- Normalized correlation

- Subpixel by second order fit to 3x3 neighborhood

- Result: 0.1 to 0.2 subpixel 


\section{Operational teams requirements}

- Know models of sensor/platform/

- Have access to complete data set

- Have continuing demands/responsibility

- Are registering same plots of land again and again - can invest effort in data preparation

- Can't take big risks on unproven methods 


\section{Know platform: Landsat team knowledge}

- Sensor geometry

- Band to band

- Sensor to platform

- Sensor to sensor

- Orbit

- Platform to Earth

- Terrain data

- DEM

- Radiometric model

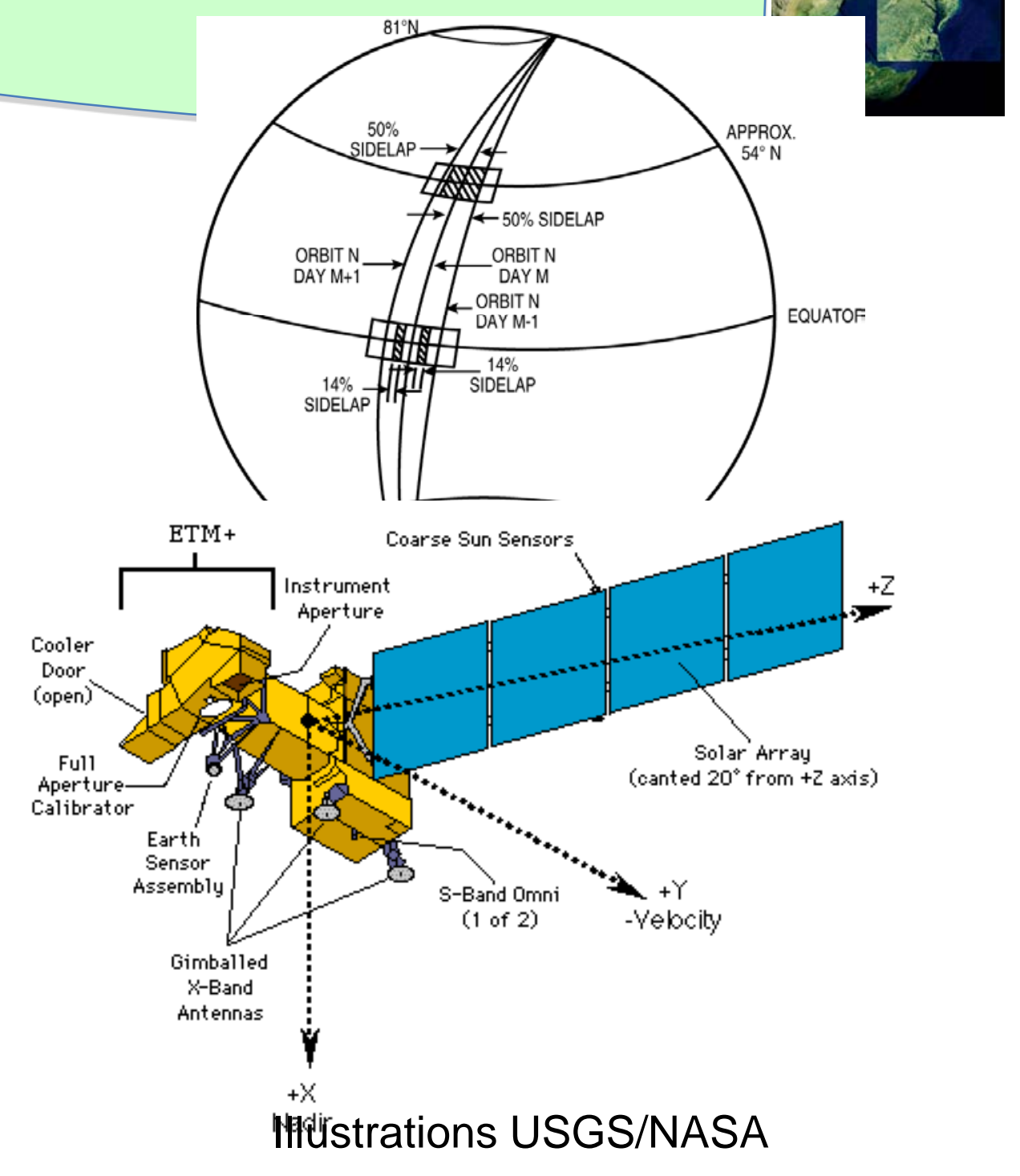




\section{Invest in data: ETM+ Chip}
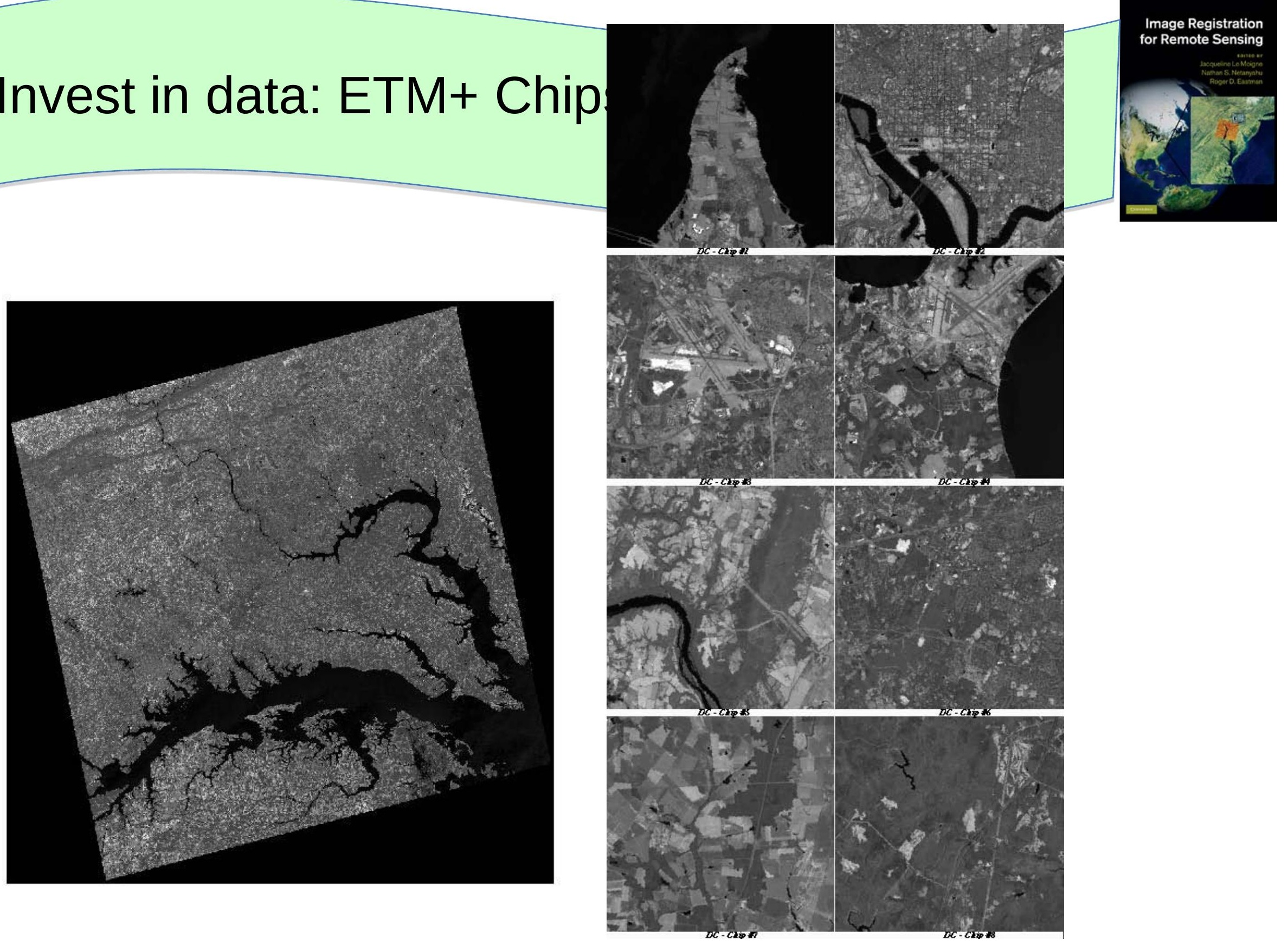
Know data: GOES channel 1 (Baja)

- Contrast reversal

day to night

- Requires use of contour matching 


\section{Use DEMs: Digital Terrain Models}

\section{Taking terrain into account in matching}

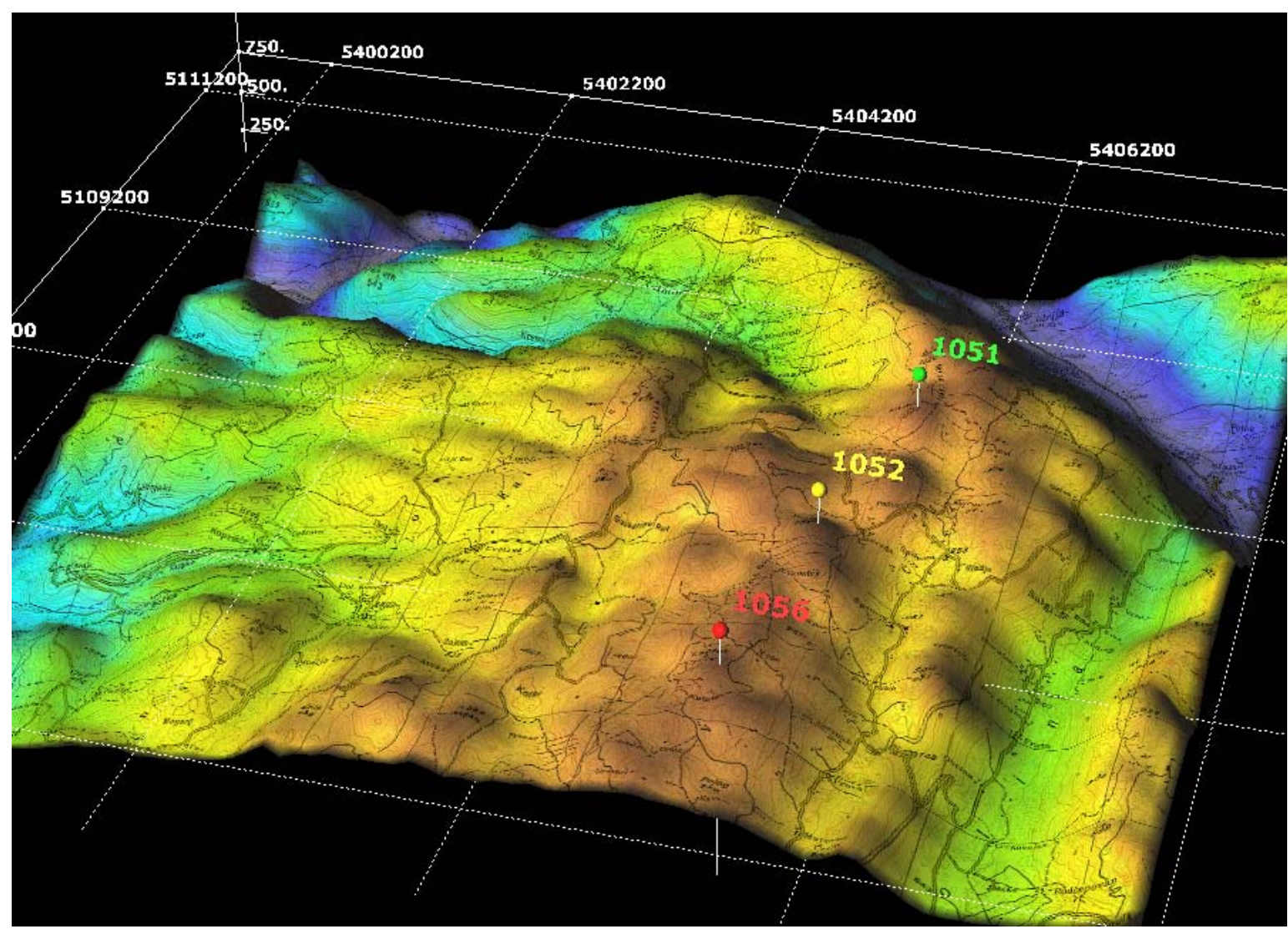


Use proven methods: Landsat 7 library

- Clean data, go fast

- Use Normalized Grey-Scale Correlation

- Missing data/gaps, need robustness

- Use Mutual Information

- Available alternative

- Use Robust Feature Matching 


\section{End Users - Earth scientists}

- Know what data is for

- Have to fuse many data sets

- Have access to ancillary data

- Know cultural and historical data

- Don't need one magic method - need toolbox of many approaches 


\section{Missouri river 1804-2002}
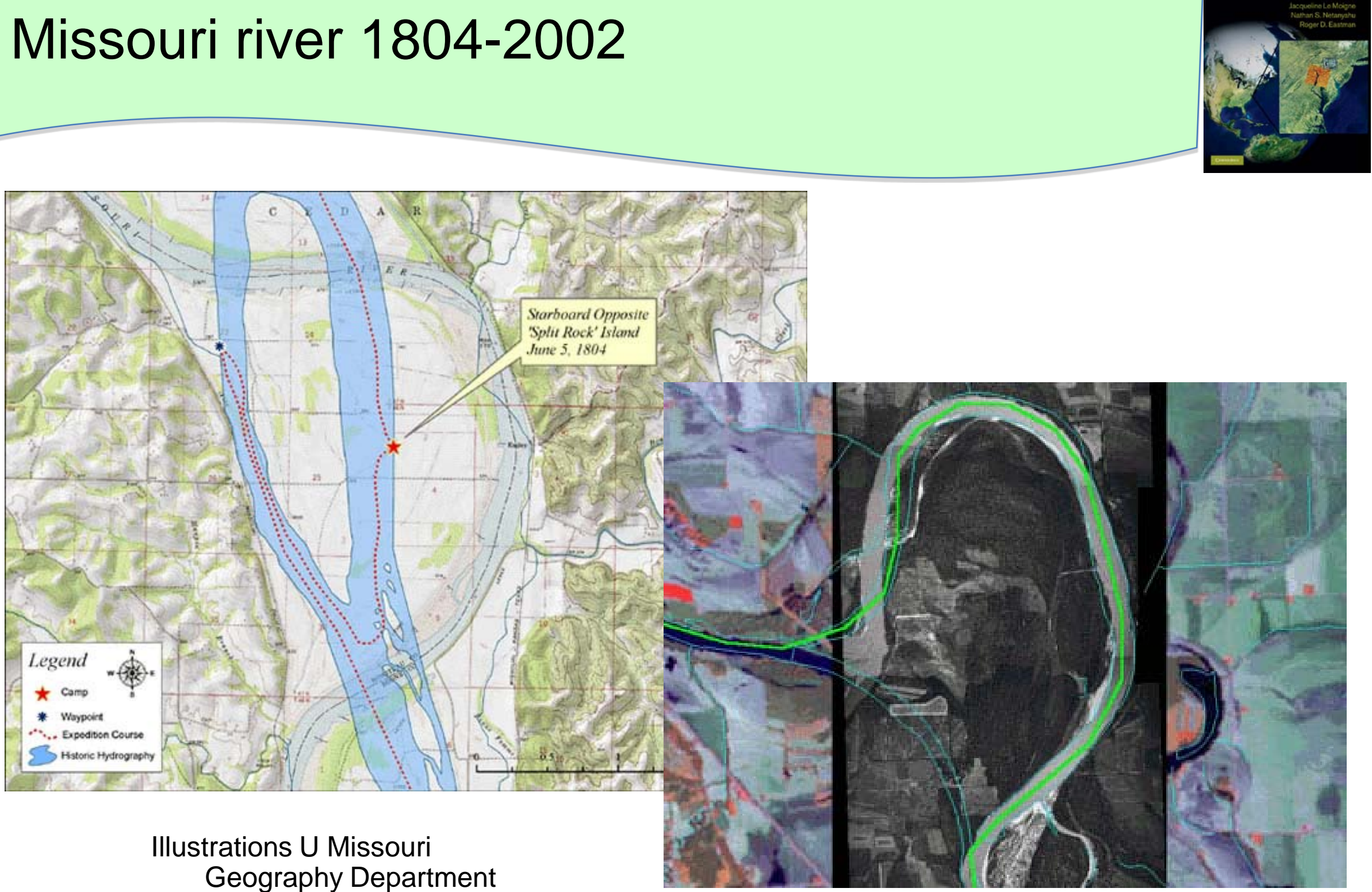


\section{Institutional challenges to "solving" IR for RS}

- Different communities/literature/requirements

- Photogrammetry

- Computer vision/image processing

- Operational teams

- Remote sensing/Earth scientists/end users

- Demanding/varying mission requirements

- Caution in system design, new methods

- Expensive sensors and images

- Hard to share data or complete models 


\title{
Conclusion
}

\author{
Jacqueline Le Moigne \\ Nathan S. Netanyahu \\ Roger D. Eastman
}




\section{THE FUTURE OF IMAGE REGISTRATION}

- Satellite sensing/imaging in full expansion

- Explosion of commercial satellites

- Exploring distant planets (Moon, Mars, etc.), e.g. Lunar Reconnaissance Orbiter (LRO)

- Future research and challenges

- Combining multiple band-to-band registrations (e.g., hyperspectral data)

- Automatically extracting windows of interest (decreasing processing time and increasing accuracy)

- Dealing with other data sources (e.g., planetary imagery, or verification of optical systems)

- Integration and fusion of multiple source imagery (various satellites, vector map, airborne, ground data, etc.)

- Onboard implementations on specialized hardware

- Multistage registration algorithms combining multiple principles and approaches and utilizing interdisciplinary systems engineering approach , thus increasing algorithms robustness and applicability 


\section{Other Memories, 1983 to 1988 ...}
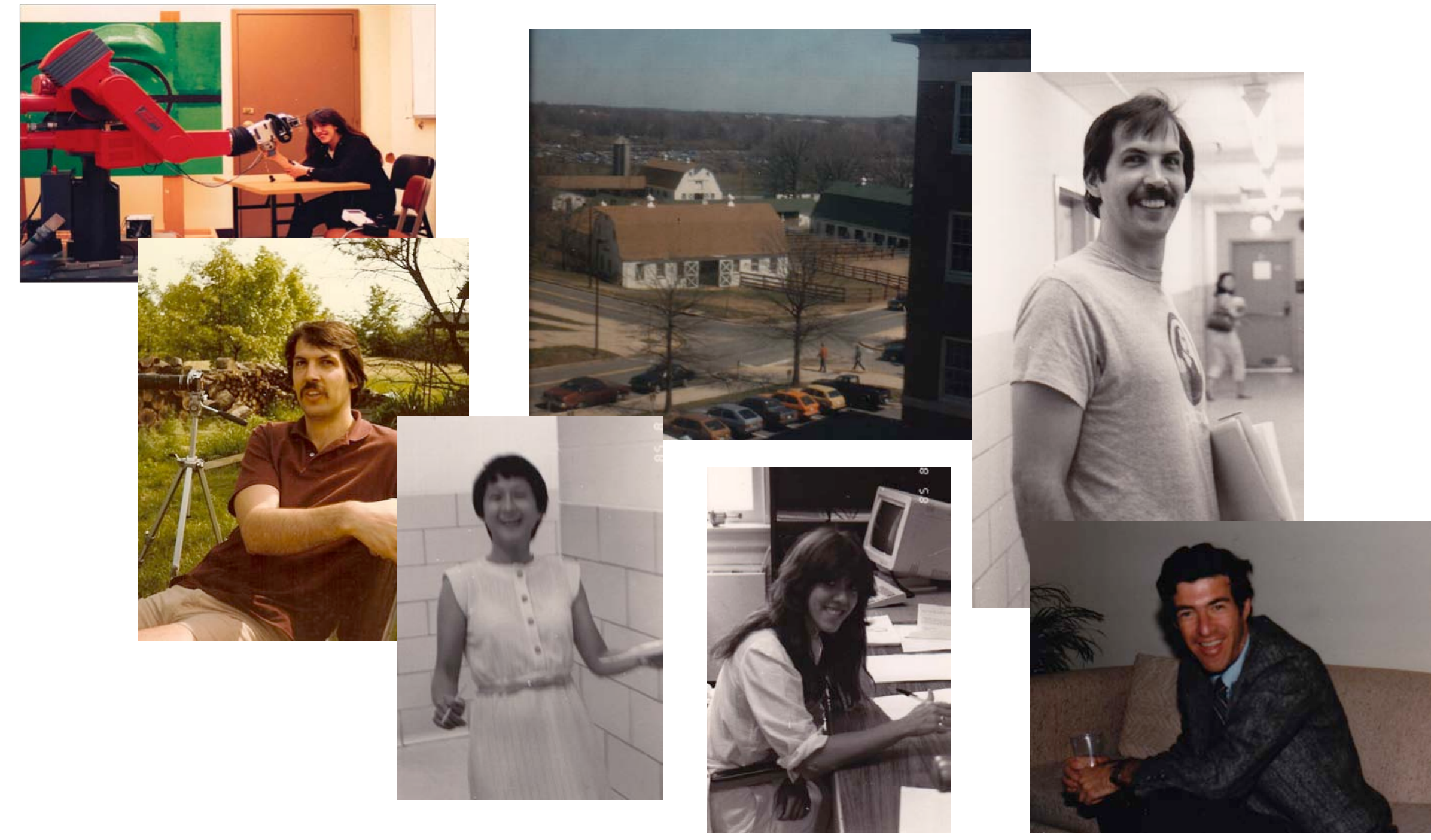


\section{The Autonomous Land Vehicle (ALV) Project}

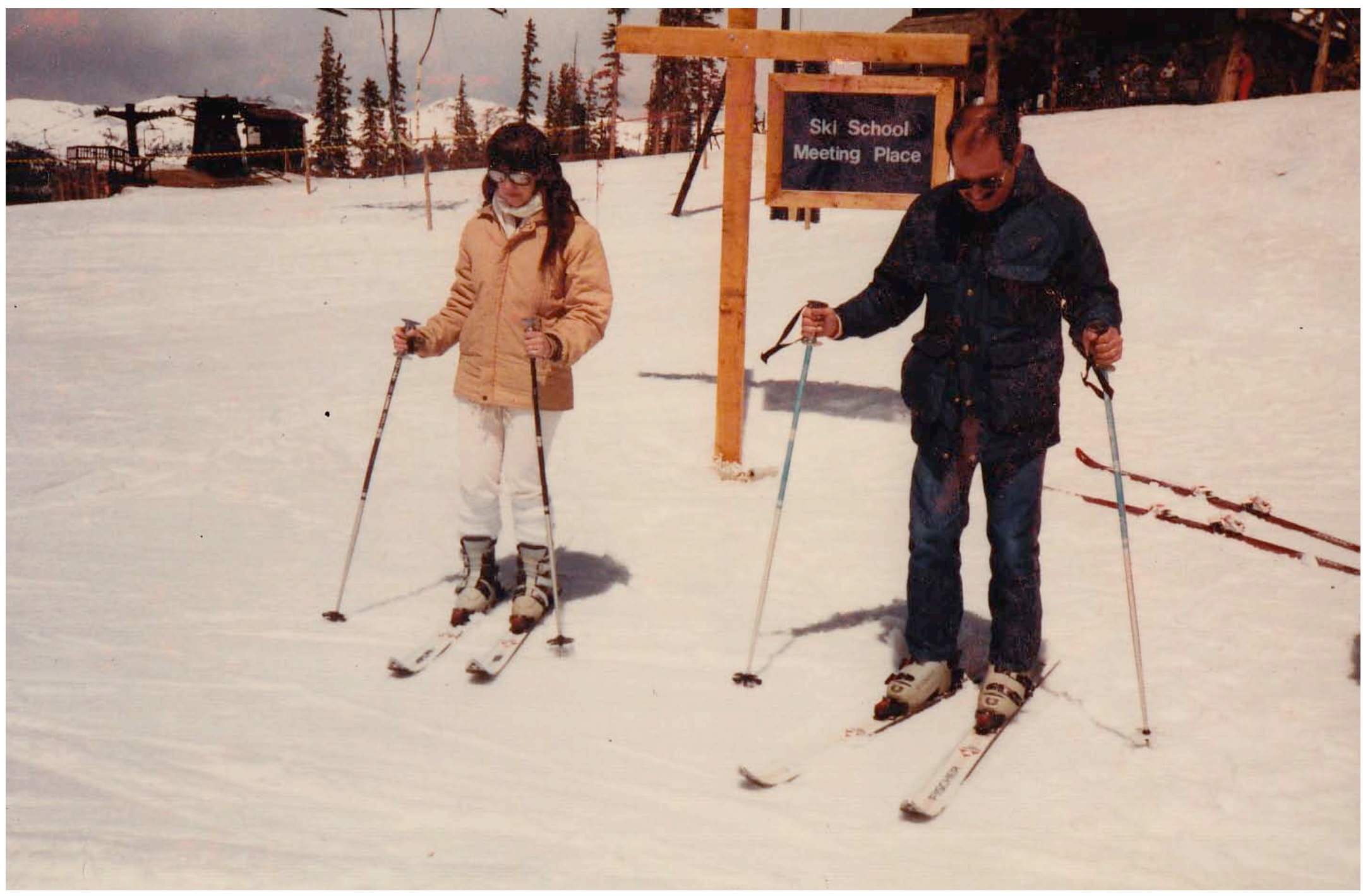


Thank You!

Jacqueline Le Moigne

Nathan S. Netanyahu

Roger D. Eastman 